\title{
A FIC-based stabilized mixed finite element method with equal order interpolation for solid-pore fluid interaction problems
}

\author{
Ignasi de-Pouplana ${ }^{\mathrm{a},{ }^{*}}$ and Eugenio Oñate ${ }^{\mathrm{a}, \mathrm{b}}$ \\ ${ }^{a}$ CIMNE - Centre Internacional de Metodes Numerics en Enginyeria, Gran \\ Capitán s/n, 08034 Barcelona, Spain \\ ${ }^{\mathrm{b}}$ Departament d'Enginyeria Civil i Ambiental (ECA), Universitat Politècnica de \\ Catalunya (UPC), Campus Nord, Edif. C1, C. Jordi Girona, 1-3, 08034 \\ Barcelona, Spain
}

May 23, 2016

\begin{abstract}
A new mixed displacement-pressure element for solving solid-pore fluid interaction problems is presented. In the resulting coupled system of equations the balance of momentum equation remains unaltered, while the mass balance equation for the pore fluid is stabilized with the inclusion of higher order terms multiplied by arbitrary dimensions in space, following the Finite Calculus (FIC) procedure. The stabilized FIC-FEM formulation can be applied to any kind of interpolation for the displacements and the pressure, but in this work we have used linear elements of equal order interpolation for both set of unknowns. Examples in 2D and 3D are presented to illustrate the accuracy of the stabilized formulation for solid-pore fluid interaction problems.
\end{abstract}

Keywords: coupled solid-pore fluid formulation, saturated soils, incompressible undrained limit, Finite Calculus, Finite Element Method.

\section{Introduction}

Problems involving the flow of a fluid through a porous medium have always been the object of special interest in geomechanics. From the classical contributions of Terzaghi [1] and Biot [2] important effort has been made in the last decades to develop competent numerical methods in poromechanics that allow analysing and understanding the complexity of the mechanisms

\footnotetext{
${ }^{*}$ Correspondence to: Ignasi de Pouplana, CIMnE, Edificio C1, Campus Nord, UPC, Gran Capitán s/n, 08034 Barcelona, Spain. Email: ipouplana@cimne.upc.edu
} 
involved in problems with a coupling between a solid skeleton and a fluid phase.

In hydrogeology, for instance, these numerical methods are important to represent the water flow through porous soil when characterizing aquifers performance (storage capacity, water transmissivity, water content, etc.), and also to study the dispersion and transport of dissolved contaminants [3].

Another important field of application is in petroleum engineering. Here, the oil-gas-soil interaction takes the leading role along with the hydraulic fracturing as a common technique to enhance reservoir permeability and well efficiency $[4,5]$.

Other applications for numerical methods in poromechanics have recently emerged for a variety of applications, such as underground storage of carbon dioxide [6], geothermal energy production simulation [7], and even analysis of interstitial flow in bone tissue [8].

In 1943, a 1D theory of consolidation was proposed by Terzaghi [1]. This theory was then extended by Biot to $3 \mathrm{D}$ cases $[2,9,10]$. Although these first works were restricted to linear elastic materials, they have been the basis for much subsequent research in geophysics, soil and rock mechanics.

The first numerical solution of Biot's formulation was obtained by Ghaboussi and Wilson [11] and the work was further developed by Zienkiewicz et al. $[12,13]$. Later on, due to the increasing interest in non-linear applications, Zienkiewicz and co-workers expanded the theory to a generalized incremental form for non-linear materials and large deformation problems $[14,15]$.

The mathematical formulation of solid skeleton and pore fluid interaction presented here is based on the model proposed by Zienkiewicz et al. [12]. The problem was originally formulated for fully saturated conditions in terms of the solid matrix displacement $u_{i}$, the mean fluid velocity relative to the solid phase $w_{i}$ and the fluid pore pressure $p$. However, in many geo-mechanical problems with no high-frequency phenomena involved, the fluid relative velocity $w_{i}$ can be neglected and so the equations can be simplified to work with only two main variables: the displacements $u_{i}$ and the pressure $p$ [16].

Although in this work we have solved a two-phase medium, i.e. soil and water, the generalization to three-phase problems, such as those encountered in unsaturated soils or in oil-gas-soil interaction, are possible extensions of the numerical approach presented here. In this regard, a simple extension of the two phase formulation to semi-saturated problems was proposed by Zienkiewicz et al. [17] and further development was reported by Gawin and Schrefler [18], and Khoei et al. [19].

In the limit of nearly incompressible pore fluid and small permeability, the coupled poromechanics formulations suffer from instability problems. Finite elements exhibit locking in the pressure field and spurious oscillations in the numerical solution for the pressure appear near this fully incompressible limit, due to the violation of the so-called Babuska-Brezzi conditions [20,21]. 
The oscillations can be overcome by locally refining the mesh and by using shape functions of the displacement field one order higher than those of the pressure field. In practical applications this is, however, not the best approach because of the increment in the computational cost. In this sense, stabilization methods have been found to be powerful tools to circumvent the Babuska-Brezzi conditions violation without compromising the efficiency of the computation.

Several stabilization techniques have been investigated in the past years in the context of computational fluid dynamics [22-27] and (incompressible) solid mechanics [28-32], and have been also extended to poromechanics problems [33-37].

Although each stabilization approach has its differential aspects, they can be classified in three main categories.

The first category comprises those techniques in which special time stepping schemes are applied in order to generate stabilization terms. Probably the earliest work in this category is due to Chorin [22] who proposed a technique to deal with incompressible fluid problems which is now referred as the fractional step method or the operator-splitting method. Such a staggered time stepping algorithm has been found to provide stabilization in the steady-state when used in a convenient form $[22,25,34,35]$.

The second type of techniques are more direct stabilization methods based on the perturbation of the fluid mass conservation equation. Thereby, instead of using special time-stepping algorithms resulting in additional terms in the steady-state approximation, the fluid balance equation is modified by adding a stabilization term multiplied by a parameter that needs to be carefully calibrated $[23,24,30,33]$. This group also includes the methods based on the concept of Polynomial-Pressure-Projections, in which the additional stabilizing terms use element-local projections of the pressure field to counteract the inherent instabilities [37-40].

The third category considered here is the Finite Increment Calculus method, also called Finite Calculus (FIC) [26,27,31,32,41,42], which is the approach also adopted in the present work. The FIC technique is based on expressing the equations of balance of mass and momentum in a space/time domain of finite size and retaining higher order terms in the Taylor series expansion typically used for expressing the change in the transported variables within the balance domain. In addition to the standard terms of infinitesimal theory, the FIC form of the balance equations contains derivatives of the classical differential equations multiplied by characteristic distances in space and/or time.

Previously stabilized FIC-FEM formulations were based on the firstorder form of the FIC balance equation in space and can be found in the literature for quasi and fully incompressible fluids and solids [26, 27, 31,32], and even for 1D and 2D poromechancis problems [43]. In this work, the more recent second-order FIC form of the mass balance equation $[41,42]$ has been 
adopted as the basis for deriving the stabilized FIC-FEM formulation for $2 \mathrm{D}$ and $3 \mathrm{D}$ poromechanics problems. This new formulation takes advantage of the second order derivatives terms to provide a simpler procedure for obtaining a residual-based stable form of the mass balance equation suitable for finite element analysis. Proof of the good results of this formulation regarding convergence and mass conservation are given in [42].

In this work we present a robust finite element for solving solid-pore fluid interaction problems, in which the continuity equation is stabilized by means of the FIC method. The element is based on a mixed displacement-pressure formulation with equal order interpolation for the displacement and pressure variables. In this work linear elements are used for simplicity.

The paper is organized as follows. First, the finite element formulation is introduced, starting from the governing equations of the problem and the fully coupled system of equations to be solved. After that, the instability problem is stated and the stabilization by means of the second-order FIC form of the mass balance equation in space is thoroughly detailed. Finally, two academic examples are solved in order to test the implemented mixed linear element and compare it to stable elements with a higher order interpolation for the displacement field.

\section{General finite element formulation}

In the theory of porous media, the effective stress is an essential concept for defining the stress state because it is responsible for the major deformations and rupture of the solid skeleton. Let $\sigma_{i j}$ be the total stress (positive in tension) acting on the total area of the soil and the pores, and $p$ the pressure of the fluid in the pores (positive in compression), the effective stress is defined as:

$$
\sigma_{i j}^{\prime}=\sigma_{i j}+\alpha p \delta_{i j}
$$

where $\delta_{i j}$ is the Kronecker delta and $\alpha$ is Biot's coefficient [44]:

$$
\alpha=1-\frac{K}{K_{s}} \leq 1
$$

with $K_{s}$ being the bulk modulus of the solid phase and $K$ the bulk modulus of the porous medium:

$$
K=\frac{E}{3(1-2 \nu)}
$$

In order to account for the coupling between the solid and fluid phases, the behaviour of a saturated porous medium is governed by the combination of two equations: the balance of momentum for the mixture solid-fluid, and the mass balance for the pore fluid. 
Balance of momentum for the mixture solid-fluid

$$
\frac{\partial \sigma_{i j}}{\partial x_{j}}+\rho b_{i}=\rho \ddot{u}_{i}
$$

where $b_{i}$ is the body force per unit mass, $\ddot{u}_{i}$ is the acceleration of the solid skeleton and $\rho$ is the density of the solid-fluid mixture $\rho=\phi \rho_{f}+(1-\phi) \rho_{s}$, being $\phi$ the porosity of the soil, $\rho_{f}$ the density of the fluid and $\rho_{s}$ the density of the solid.

Let us introduce the general definition for the effective stress (1) into the balance of momentum equation (4). This gives

$$
\frac{\partial}{\partial x_{j}}\left(\sigma_{i j}^{\prime}-\alpha p \delta_{i j}\right)+\rho b_{i}-\rho \ddot{u}_{i}=0
$$

Mass balance for the pore fluid

$$
\frac{\partial \zeta}{\partial t}+\frac{\partial q_{i}}{\partial x_{i}}=0
$$

where $\zeta$ is the fluid mass content per unit volume and $q_{i}$ represents the flow rate. It should be noted that Equation (6) is in a linearized form of the general mass balance expression as the fluid density variation effect has been ignored.

The mass balance equation is modified as follows. Let us first consider the constitutive equation presented in [2] relating the fluid mass content per unit volume $\zeta$ with the volumetric strain of the solid skeleton $\epsilon$ and the pore pressure $p$.

$$
\zeta=\alpha \epsilon+\frac{p}{Q}
$$

where $Q$ is a combined compressibility of the fluid-solid phases, also called Biot's modulus [44]

$$
\frac{1}{Q}=\frac{\alpha-\phi}{K_{s}}+\frac{\phi}{K_{f}}
$$

being $K_{f}$ the bulk modulus of the fluid phase.

Let us now make use of the generalized form of Darcy's law

$$
q_{i}=-\frac{1}{\mu} k_{i j}\left(\frac{\partial p}{\partial x_{j}}-\rho_{f} b_{j}\right)
$$

where $\mu$ is the dynamic viscosity of the fluid and $k_{i j}$ is the intrinsic permeability matrix of the porous medium.

Taking into account the relations from equations (7) and (9), the mass balance equation (6) can be rewritten as

$$
\alpha \dot{\epsilon}+\frac{\dot{p}}{Q}+\frac{\partial}{\partial x_{i}}\left[-\frac{1}{\mu} k_{i j}\left(\frac{\partial p}{\partial x_{j}}-\rho_{f} b_{j}\right)\right]=0
$$


Equations (5) and (10) have to be supplemented by a constitutive law for the solid skeleton. In general, for any non-linear material we may consider an incremental form with a tangential constitutive tensor $D_{i j k l}^{t a n}$ as

$$
d \sigma_{i j}^{\prime}=D_{i j k l}^{t a n} d \varepsilon_{k l}
$$

with

$$
\varepsilon_{i j}=\frac{1}{2}\left(\frac{\partial u_{i}}{\partial x_{j}}+\frac{\partial u_{j}}{\partial x_{i}}\right)
$$

The boundary conditions for this problem are specified as:

$$
\begin{aligned}
u_{i} & =\tilde{u}_{i} \quad \text { on } \quad \Gamma_{u} \\
\left(\sigma_{i j}^{\prime}-\alpha p \delta_{i j}\right) n_{j} & =\tilde{t}_{i} \quad \text { on } \quad \Gamma_{t}
\end{aligned}
$$

where $\tilde{u}_{i}$ and $\tilde{t}_{i}$ are the prescribed displacements and surface tractions, respectively.

$$
\begin{aligned}
p & =\tilde{p} \quad \text { on } \quad \Gamma_{p} \\
{\left[-\frac{1}{\mu} k_{i j}\left(\frac{\partial p}{\partial x_{j}}-\rho_{f} b_{j}\right)\right] n_{i} } & =\tilde{q}_{n} \quad \text { on } \quad \Gamma_{q}
\end{aligned}
$$

where $\tilde{p}$ and $\tilde{q}_{n}$ are the prescribed pore pressure and the normal flow rate, respectively.

In order to express the resultant system of equations in a more compact manner, we will rewrite equations (5) and (10) in matrix form using Voigt notation as follows.

- Balance of momentum

$$
\boldsymbol{S}^{T}\left(\boldsymbol{\sigma}^{\prime}-\alpha p \boldsymbol{m}\right)+\rho \boldsymbol{b}-\rho \ddot{\boldsymbol{u}}=\mathbf{0}
$$

- Mass balance

$$
\alpha \boldsymbol{m}^{T} \boldsymbol{S} \dot{\boldsymbol{u}}+\frac{\dot{p}}{Q}+\boldsymbol{\nabla}^{T}\left[-\frac{1}{\mu} \boldsymbol{k}\left(\boldsymbol{\nabla} p-\rho_{f} \boldsymbol{b}\right)\right]=0
$$

For a general 3D case, we have

$$
\boldsymbol{S}=\left[\begin{array}{ccc}
\frac{\partial}{\partial x} & 0 & 0 \\
0 & \frac{\partial}{\partial y} & 0 \\
0 & 0 & \frac{\partial}{\partial z} \\
\frac{\partial}{\partial y} & \frac{\partial}{\partial x} & 0 \\
0 & \frac{\partial}{\partial z} & \frac{\partial}{\partial y} \\
\frac{\partial}{\partial z} & 0 & \frac{\partial}{\partial x}
\end{array}\right] \quad ; \quad \nabla=\left[\frac{\partial}{\partial x}, \frac{\partial}{\partial y}, \frac{\partial}{\partial z}\right]^{T}
$$




$$
\begin{gathered}
\boldsymbol{\sigma}^{\prime}=\left[\begin{array}{llllll}
\sigma_{x x}^{\prime}, & \sigma_{y y}^{\prime}, & \sigma_{z z}^{\prime}, & \sigma_{x y}^{\prime}, & \sigma_{y z}^{\prime}, & \sigma_{z x}^{\prime}
\end{array}\right]^{T} \\
\boldsymbol{m}=\left[\begin{array}{llllll}
1, & 1, & 1, & 0, & 0, & 0
\end{array}\right]^{T}
\end{gathered}
$$

The above system of partial differential equations (17) and (18) can be discretized by interpolating the displacement and pressure fields as: $\boldsymbol{u}=$ $\boldsymbol{N}_{u} \overline{\boldsymbol{u}}$ and $p=\boldsymbol{N}_{p} \overline{\boldsymbol{p}}$ where (.âĂ $\left.c ́\right)$ denotes nodal values and

$$
\begin{gathered}
\boldsymbol{N}_{u}=\left[\begin{array}{cccccccccc}
\hat{N}_{1} & 0 & 0 & \hat{N}_{2} & 0 & 0 & \ldots & \hat{N}_{\hat{n}} & 0 & 0 \\
0 & \hat{N}_{1} & 0 & 0 & \hat{N}_{2} & 0 & \ldots & 0 & \hat{N}_{\hat{n}} & 0 \\
0 & 0 & \hat{N}_{1} & 0 & 0 & \hat{N}_{2} & \ldots & 0 & 0 & \hat{N}_{\hat{n}}
\end{array}\right] \\
\boldsymbol{N}_{p}=\left[N_{1}, N_{2}, \ldots, N_{n}\right]
\end{gathered}
$$

with $\hat{N}_{i}$ and $N_{i}$ being, respectively, the shape functions of the displacement and pressure interpolations, which do not necessarily need to coincide.

Following the standard Galerkin technique $[45,46]$, we left multiply Equation (17) by test functions $\boldsymbol{N}_{u}^{T}$ and Equation (18) by test functions $\boldsymbol{N}_{p}^{T}$ and integrate them over the spatial domain $\Omega$ to obtain the following set of ordinary differential equations

- Balance of momentum

$$
\boldsymbol{r}_{u}:=\boldsymbol{M} \ddot{\overline{\boldsymbol{u}}}+\int_{\Omega} \boldsymbol{B}^{T} \boldsymbol{\sigma}^{\prime} d \Omega-\boldsymbol{Q} \overline{\boldsymbol{p}}-\boldsymbol{f}_{u}=\mathbf{0}
$$

- Mass balance

$$
\boldsymbol{r}_{p}:=\boldsymbol{Q}^{T} \dot{\overline{\boldsymbol{u}}}+\boldsymbol{C} \dot{\overline{\boldsymbol{p}}}+\boldsymbol{H} \overline{\boldsymbol{p}}-\boldsymbol{f}_{p}=\mathbf{0}
$$

where $\boldsymbol{r}_{u}$ and $\boldsymbol{r}_{p}$ are the residual vectors for the momentum and the mass balance equation, and

$$
\begin{gathered}
\boldsymbol{M}=\int_{\Omega} \boldsymbol{N}_{u}^{T} \rho \boldsymbol{N}_{u} d \Omega \quad ; \quad \boldsymbol{Q}=\int_{\Omega} \boldsymbol{B}^{T} \alpha \boldsymbol{m} \boldsymbol{N}_{p} d \Omega \\
\boldsymbol{C}=\int_{\Omega} \boldsymbol{N}_{p}^{T} \frac{1}{Q} \boldsymbol{N}_{p} d \Omega ; \boldsymbol{H}=\int_{\Omega}\left(\boldsymbol{\nabla} \boldsymbol{N}_{p}\right)^{T} \frac{1}{\mu} \boldsymbol{k} \boldsymbol{\nabla} \boldsymbol{N}_{p} d \Omega \\
\boldsymbol{f}_{u}=\int_{\Omega} \boldsymbol{N}_{u}^{T} \rho \boldsymbol{b} d \Omega+\int_{\Gamma_{t}} \boldsymbol{N}_{u}^{T} \tilde{\boldsymbol{t}} d \Gamma \\
\boldsymbol{f}_{p}=\int_{\Omega}\left(\boldsymbol{\nabla} \boldsymbol{N}_{p}\right)^{T} \frac{1}{\mu} \boldsymbol{k} \rho_{f} \boldsymbol{b} d \Omega-\int_{\Gamma_{q}} \boldsymbol{N}_{p}^{T} \tilde{q}_{n} d \Gamma
\end{gathered}
$$

with $\boldsymbol{B}=\boldsymbol{S} \boldsymbol{N}_{u}$. 
The time derivatives of $\boldsymbol{u}$ and $p$ are approximated using the Generalized Newmark scheme. Thus, for a new time step $n+1$, we use the GN22 scheme for displacements [47]:

$$
\begin{gathered}
\boldsymbol{u}_{n+1}=\boldsymbol{u}_{n}+\Delta t \dot{\boldsymbol{u}}_{n}+\left(\frac{1}{2}-\beta\right) \Delta t^{2} \ddot{\boldsymbol{u}}_{n}+\beta \Delta t^{2} \ddot{\boldsymbol{u}}_{n+1} \\
\dot{\boldsymbol{u}}_{n+1}=\dot{\boldsymbol{u}}_{n}+(1-\gamma) \Delta t \ddot{\boldsymbol{u}}_{n}+\gamma \Delta t \ddot{\boldsymbol{u}}_{n+1}
\end{gathered}
$$

and the GN11 scheme for pressure:

$$
p_{n+1}=p_{n}+(1-\theta) \Delta t \dot{p}_{n}+\theta \Delta t \dot{p}_{n+1}
$$

which are unconditionally stable for $\beta \geq \frac{1}{4}, \gamma \geq \frac{1}{2}$ and $\theta \geq \frac{1}{2}$ [48].

The residual vectors for the momentum and mass balance equations can be rewritten as

$$
\begin{aligned}
& \left(\boldsymbol{r}_{u}\right)_{n+1}=\boldsymbol{M}\left[\frac{\overline{\boldsymbol{u}}_{n+1}-\hat{\overline{\boldsymbol{u}}}_{n}}{\beta \Delta t^{2}}\right]+\left(\int_{\Omega} \boldsymbol{B}^{T} \boldsymbol{\sigma}^{\prime} d \Omega\right)_{n+1}-\boldsymbol{Q} \overline{\boldsymbol{p}}_{n+1}-\left(\boldsymbol{f}_{u}\right)_{n+1} \\
& \left(\boldsymbol{r}_{p}\right)_{n+1}=\boldsymbol{Q}^{T}\left[\frac{\gamma \overline{\boldsymbol{u}}_{n+1}}{\beta \Delta t}+\hat{\overline{\boldsymbol{u}}}_{n}\right]+\boldsymbol{C}\left[\frac{\overline{\boldsymbol{p}}_{n+1}}{\theta \Delta t}+\hat{\overline{\boldsymbol{p}}}_{n}\right]+\boldsymbol{H} \overline{\boldsymbol{p}}_{n+1}-\left(\boldsymbol{f}_{p}\right)_{n+1}
\end{aligned}
$$

where $\overline{\boldsymbol{u}}_{n+1}$ and $\overline{\boldsymbol{p}}_{n+1}$ are the nodal unknowns at time $n+1$, and $\hat{\overline{\boldsymbol{u}}}_{n}, \hat{\overline{\boldsymbol{u}}}_{n}$ and $\hat{\overline{\boldsymbol{p}}}_{n}$ stand for values that are computed from known parameters at time $n$ as:

$$
\begin{aligned}
& \hat{\overline{\boldsymbol{u}}}_{n}=\overline{\boldsymbol{u}}_{n}+\Delta t \dot{\overline{\boldsymbol{u}}}_{n}+\left(\frac{1}{2}-\beta\right) \Delta t^{2} \ddot{\overline{\boldsymbol{u}}}_{n} \\
& \hat{\overline{\overline{\boldsymbol{u}}}}_{n}=\dot{\overline{\boldsymbol{u}}}_{n}+(1-\gamma) \Delta t \ddot{\overline{\boldsymbol{u}}}_{n}-\frac{\gamma}{\beta \Delta t} \hat{\overline{\boldsymbol{u}}}_{n} \\
& \hat{\overline{\dot{p}}}_{n}=\frac{\theta-1}{\theta} \dot{\overline{\boldsymbol{p}}}_{n}-\frac{1}{\theta \Delta t} \overline{\boldsymbol{p}}_{n}
\end{aligned}
$$

The Newton-Raphson method is used to solve the problem iteratively. Thus, at a time step $n+1$ and the iteration $i+1$ we have:

$$
\begin{aligned}
& {\left[\begin{array}{ll}
\frac{\partial \boldsymbol{r}_{u}}{\partial \overline{\boldsymbol{u}}} & \frac{\partial \boldsymbol{r}_{u}}{\partial \overline{\boldsymbol{p}}} \\
\frac{\partial \boldsymbol{r}_{p}}{\partial \overline{\boldsymbol{u}}} & \frac{\partial \boldsymbol{r}_{p}}{\partial \overline{\boldsymbol{p}}}
\end{array}\right]_{n+1}^{i}\left[\begin{array}{l}
\delta \overline{\boldsymbol{u}} \\
\delta \overline{\boldsymbol{p}}
\end{array}\right]_{n+1}^{i+1}=-\left[\begin{array}{l}
\boldsymbol{r}_{u} \\
\boldsymbol{r}_{p}
\end{array}\right]_{n+1}^{i}} \\
& {\left[\begin{array}{cc}
\frac{1}{\beta \Delta t^{2}} \boldsymbol{M}+\boldsymbol{K}_{\text {tan }} & -\boldsymbol{Q} \\
\frac{\gamma}{\beta \Delta t} \boldsymbol{Q}^{T} & \frac{1}{\theta \Delta t} \boldsymbol{C}+\boldsymbol{H}
\end{array}\right]_{n+1}^{i}\left[\begin{array}{l}
\delta \overline{\boldsymbol{u}} \\
\delta \overline{\boldsymbol{p}}
\end{array}\right]_{n+1}^{i+1}=-\left[\begin{array}{l}
\boldsymbol{r}_{u} \\
\boldsymbol{r}_{p}
\end{array}\right]_{n+1}^{i}}
\end{aligned}
$$

where $\boldsymbol{K}_{\text {tan }}$ is the tangent stiffness matrix:

$$
\boldsymbol{K}_{\text {tan }}=\frac{\partial}{\partial \overline{\boldsymbol{u}}} \int_{\Omega} \boldsymbol{B}^{T} \boldsymbol{\sigma}^{\prime} d \Omega=\int_{\Omega} \boldsymbol{B}^{T} \boldsymbol{D}_{\tan } \boldsymbol{B} d \Omega
$$

Note that the system of equations (38) can be made symmetric by multiplying Equation (33) by $-\beta \Delta t / \gamma$. 


\section{$3 \quad$ FIC stabilized element}

\subsection{Introduction to the FIC-stabilization procedure}

For a quasi-static problem the term involving the mass matrix is omitted. Also, in the undrained-incompressible limit, i.e. when $\boldsymbol{k} \rightarrow \mathbf{0}$ and $Q \rightarrow \infty$, the matrices $\boldsymbol{C}$ and $\boldsymbol{H}$ vanish and the system of equations (38) becomes

$$
\left[\begin{array}{cc}
\boldsymbol{K}_{\tan } & -\boldsymbol{Q} \\
-\boldsymbol{Q}^{T} & \mathbf{0}
\end{array}\right]_{n+1}^{i}\left[\begin{array}{l}
\delta \overline{\boldsymbol{u}} \\
\delta \overline{\boldsymbol{p}}
\end{array}\right]_{n+1}^{i+1}=-\left[\begin{array}{c}
\boldsymbol{r}_{u} \\
\boldsymbol{r}_{p}^{*}
\end{array}\right]_{n+1}^{i}
$$

The resultant system matrix is almost identical to that frequently encountered in the solution of incompressible elasticity or incompressible fluid mechanics problems $[24,32]$. In such cases, the spaces used to approximate the displacement (or the velocity) and pressure fields must fulfill the Babuska-Brezzi conditions [20,21] or the Zienkiewicz-Taylor patch test $[49,50]$ in order to avoid spurious oscillations and locking in the pressure field.

With the equations presented so far, these conditions can be accomplished using shape functions for the displacement field one order higher than those for the pressure field. Some examples of "stable" 2D elements are depicted in Figure 1.

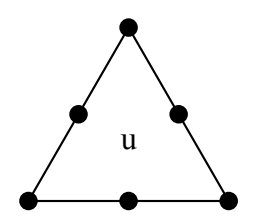

(a) Quadratic displacement and linear pressure.

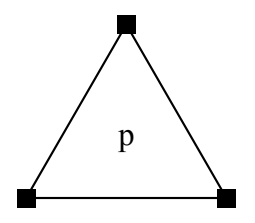

(b)

(b) Biquadratic displacement and bilinear pressure.

Figure 1: Some 2D elements that fulfill the Babuska-Brezzi conditions.

However, low order finite elements with equal order interpolation for the displacements and the pressure are very attractive due to their simplicity and efficiency, and so stabilization techniques must be applied if one aims at solving large scale 3D computations.

The stabilization approach implemented in this work relies on the Finite Calculus (FIC) method [26, 27,31,32,41,42] and affects only the continuity equation with the balance of momentum remaining unchanged.

For the sake of clarity, let us redefine the mass balance equation (10) as:

$$
r_{p}:=\alpha \dot{\epsilon}+\frac{\dot{p}}{Q}+\frac{\partial}{\partial x_{j}}\left[-\frac{1}{\mu} k_{j k}\left(\frac{\partial p}{\partial x_{k}}-\rho_{f} b_{k}\right)\right]=0
$$


As stated in the introduction, this technique is based on the second-order FIC form of the mass balance equation in space for a quasi-incompressible fluid. This can be written as [42]:

$$
r_{p}+\frac{h_{i}^{2}}{12} \frac{\partial^{2} r_{p}}{\partial x_{i}^{2}}=0 \quad \text { in } \Omega \quad i=1, n_{s}
$$

where $n_{s}$ are the number of space dimensions (i.e. $n_{s}=3$ for $3 \mathrm{D}$ problems).

In a $2 \mathrm{D}$ case Equation (42) is obtained by expressing the balance of mass in a rectangular domain of finite size with dimensions $h_{1} \times h_{2}$, where $h_{i}$ are arbitrary distances, and retaining up to third-order terms in the Taylor series expansions used for expressing the change of mass within the balance domain [41]. The derivation of Equation (42) for a 1D problem is shown in [42].

The FIC term in Equation (42) plays the role of a space stabilization term, in which $h_{i}$ are space dimensions related to the characteristic element dimensions. Note that for $h_{i} \rightarrow 0$ the standard form of the mass balance equation (41), as given by the infinitesimal theory, is recovered.

Remark 1: Equation (42) can be interpreted as a particular class of residualbased stabilization methods applied to the strong form of the mass conservation equation. This ensures the consistency of the stabilization method in the discretized problem.

\subsection{Modified FIC-stabilized form of the mass balance equa- tion}

Let us first expand Equation (42) using Equation (41) as

$$
\begin{aligned}
& \alpha \dot{\epsilon}+\frac{1}{Q} \dot{p}+\frac{\partial}{\partial x_{j}}\left[-\frac{1}{\mu} k_{j k}\left(\frac{\partial p}{\partial x_{k}}-\rho_{f} b_{k}\right)\right]+\frac{h_{i}^{2}}{12} \frac{\partial^{2}}{\partial x_{i}^{2}}(\alpha \dot{\epsilon}) \\
& +\frac{h_{i}^{2}}{12} \frac{\partial^{2}}{\partial x_{i}^{2}}\left(\frac{1}{Q} \dot{p}\right)+\frac{h_{i}^{2}}{12} \frac{\partial^{2}}{\partial x_{i}^{2}}\left\{\frac{\partial}{\partial x_{j}}\left[-\frac{1}{\mu} k_{j k}\left(\frac{\partial p}{\partial x_{k}}-\rho_{f} b_{k}\right)\right]\right\}=0
\end{aligned}
$$

Next we split the stress tensor into its deviatoric and volumetric components as

$$
\sigma_{i j}=s_{i j}+\sigma \delta_{i j}
$$

where $s_{i j}$ is the deviatoric stress tensor and $\sigma$ is the hydrostatic pressure defined as $\sigma=\sigma_{i i} / 3$.

In the same way, we split the strain tensor into its deviatoric and volumetric parts as

$$
\varepsilon_{i j}=e_{i j}+\frac{1}{3} \epsilon \delta_{i j}
$$

with $e_{i j}$ being the deviatoric strain tensor and $\epsilon=\varepsilon_{i i}$ the volumetric dilation. 
Now let us write the isotropic linear elastic constitutive equations:

$$
s_{i j}=2 G e_{i j}
$$

where $G$ is the shear modulus,

$$
\sigma=\sigma^{\prime}-\alpha p
$$

where $\sigma^{\prime}=\sigma_{i i}^{\prime} / 3$ is the mean effective stress and $\alpha$ is the Biot's coefficient defined in Equation (2).

Combining Equations (44), (45), (46) and (47), we express the stress tensor as

$$
\sigma_{i j}=2 G \varepsilon_{i j}-\frac{2 G}{3} \epsilon \delta_{i j}+\sigma^{\prime} \delta_{i j}-\alpha p \delta_{i j}
$$

At this point, we substitute the above expression in the balance of momentum (4). This gives

$$
\frac{\partial}{\partial x_{j}}\left(2 G \varepsilon_{i j}\right)-\frac{\partial}{\partial x_{i}}\left(\frac{2 G}{3} \epsilon\right)+\frac{\partial \sigma^{\prime}}{\partial x_{i}}-\frac{\partial}{\partial x_{i}}(\alpha p)+\rho b_{i}-\rho \ddot{u}_{i}=0
$$

From this point forward, in the derivation of equations (43), (49) and in the following, we neglect the space and time changes of $\alpha, Q, G$ and $\rho$ in the derivatives.

If we now apply the divergence operator to both sides of Equation (49), differentiate it with respect to time, and rearrange the terms we can obtain

$$
\frac{\partial^{2} \dot{\epsilon}}{\partial x_{i}^{2}}=\frac{3}{2 G}\left[2 G \frac{\partial^{2} \dot{\varepsilon}_{i j}}{\partial x_{i} \partial x_{j}}+\frac{\partial^{2} \dot{\sigma}^{\prime}}{\partial x_{i}^{2}}-\alpha \frac{\partial^{2} \dot{p}}{\partial x_{i}^{2}}+\rho \frac{\partial \dot{b}_{i}}{\partial x_{i}}-\rho \frac{D}{D t}\left(\frac{\partial \ddot{u}_{i}}{\partial x_{i}}\right)\right]
$$

In the previous equation the term $\rho \frac{\partial \dot{b}_{i}}{\partial x_{i}}$ can be neglected and the term involving the third derivative of the displacements with respect to time can be obtained from the mass balance equation. Thus if we differentiate Equation (10) twice with respect to time assuming that the time derivative of the body force per unit mass is negligible, and we take into account the definition of the volumetric strain $\epsilon=\frac{\partial u_{i}}{\partial x_{i}}$, we obtain

$$
\frac{D}{D t}\left(\frac{\partial \ddot{u}_{i}}{\partial x_{i}}\right)=\frac{1}{\alpha}\left[\frac{\partial}{\partial x_{j}}\left(\frac{1}{\mu} k_{j k} \frac{\partial \ddot{p}}{\partial x_{k}}\right)-\frac{1}{Q} \frac{D \ddot{p}}{D t}\right]
$$

Introducing (51) into (50) gives

$$
\begin{aligned}
\frac{\partial^{2} \dot{\epsilon}}{\partial x_{i}^{2}}= & \frac{3}{2 G}\left\{2 G \frac{\partial^{2} \dot{\varepsilon}_{i j}}{\partial x_{i} \partial x_{j}}+\frac{\partial^{2} \dot{\sigma}^{\prime}}{\partial x_{i}^{2}}-\alpha \frac{\partial^{2} \dot{p}}{\partial x_{i}^{2}}\right. \\
& \left.-\frac{\rho}{\alpha}\left[\frac{\partial}{\partial x_{j}}\left(\frac{1}{\mu} k_{j k} \frac{\partial \ddot{p}}{\partial x_{k}}\right)-\frac{1}{Q} \frac{D \ddot{p}}{D t}\right]\right\}
\end{aligned}
$$


At this point, we can already substitute the above relation in Equation (43) giving

$$
\begin{aligned}
& \alpha \dot{\epsilon}+\frac{1}{Q} \dot{p}+\frac{\partial}{\partial x_{j}}\left[-\frac{1}{\mu} k_{j k}\left(\frac{\partial p}{\partial x_{k}}-\rho_{f} b_{k}\right)\right]+\frac{h_{i}^{2} \alpha}{8 G}\left\{2 G \frac{\partial^{2} \dot{\varepsilon}_{i j}}{\partial x_{i} \partial x_{j}}\right. \\
& \left.+\frac{\partial^{2} \dot{\sigma}^{\prime}}{\partial x_{i}^{2}}-\alpha \frac{\partial^{2} \dot{p}}{\partial x_{i}^{2}}-\frac{\rho}{\alpha}\left[\frac{\partial}{\partial x_{j}}\left(\frac{1}{\mu} k_{j k} \frac{\partial \ddot{p}}{\partial x_{k}}\right)-\frac{1}{Q} \frac{D \ddot{p}}{D t}\right]\right\}+\frac{h_{i}^{2}}{12} \frac{\partial^{2}}{\partial x_{i}^{2}}\left(\frac{1}{Q} \dot{p}\right) \\
& +\frac{h_{i}^{2}}{12} \frac{\partial^{2}}{\partial x_{i}^{2}}\left\{\frac{\partial}{\partial x_{j}}\left[-\frac{1}{\mu} k_{j k}\left(\frac{\partial p}{\partial x_{k}}-\rho_{f} b_{k}\right)\right]\right\}=0
\end{aligned}
$$

The above equation can be simplified if we take into account that the last term involves the fourth order spatial derivative of the pore pressure and the third order spatial derivative of the body force per unit mass. In practical problems these terms are either zero (for constant body forces) or much smaller than the others. Hence, these terms will be omitted for the rest of this work. Furthermore, numerical results have shown that the terms $\frac{\partial}{\partial x_{j}}\left(\frac{1}{\mu} k_{j k} \frac{\partial \ddot{p}}{\partial x_{k}}\right)$ and $\frac{1}{Q} \frac{D \ddot{p}}{D t}$ can be neglected without loss of accuracy. Thereby, Equation (53) is written in the simpler form as

$$
\begin{aligned}
& \alpha \dot{\epsilon}+\frac{1}{Q} \dot{p}+\frac{\partial}{\partial x_{j}}\left[-\frac{1}{\mu} k_{j k}\left(\frac{\partial p}{\partial x_{k}}-\rho_{f} b_{k}\right)\right] \\
& +\frac{h_{i}^{2} \alpha}{8 G}\left\{2 G \frac{\partial^{2} \dot{\varepsilon}_{i j}}{\partial x_{i} \partial x_{j}}+\frac{\partial^{2} \dot{\sigma}^{\prime}}{\partial x_{i}^{2}}-\alpha \frac{\partial^{2} \dot{p}}{\partial x_{i}^{2}}\right\}+\frac{h_{i}^{2}}{12} \frac{\partial^{2}}{\partial x_{i}^{2}}\left(\frac{1}{Q} \dot{p}\right)=0
\end{aligned}
$$

In the following we will assume $h_{i}=h$ where $h$ is a characteristic length related to a typical average dimension of each element in the mesh. After rearranging terms, Equation (54) can be rewritten as

$$
\begin{aligned}
& \alpha \dot{\epsilon}+\frac{1}{Q} \dot{p}+\frac{\partial}{\partial x_{i}}\left[-\frac{1}{\mu} k_{i j}\left(\frac{\partial p}{\partial x_{j}}-\rho_{f} b_{j}\right)\right] \\
& +\tau \frac{\partial}{\partial x_{i}}\left[2 G \frac{\partial \dot{\varepsilon}_{i j}}{\partial x_{j}}+\frac{\partial \dot{\sigma}^{\prime}}{\partial x_{i}}-\left(\alpha-\frac{2 G}{3 \alpha Q}\right) \frac{\partial \dot{p}}{\partial x_{i}}\right]=0
\end{aligned}
$$

where $\tau$ is a stabilization parameter given by

$$
\tau=\frac{h^{2} \alpha}{8 G}
$$

The form of the stabilization parameter in Equation (56) resembles that typically used in other stabilized procedures. We note that this term has naturally formed from the FIC formulation.

Remark 2: In the examples solved in the work we have chosen $h=l^{e}$, where $l^{e}$ is a characteristic element length that, for $2 \mathrm{D}$ problems, is taken as the 
diameter of a circle with the area of the element, while for 3D problems, it is the diameter of a sphere with the volume of the element. In essence

$$
\begin{aligned}
& l^{e}=\sqrt{\frac{4 A^{e}}{\pi}} \text { in } 2 \mathrm{D} \\
& l^{e}=\sqrt[3]{\frac{6 V^{e}}{\pi}} \text { in } 3 \mathrm{D}
\end{aligned}
$$

where $A^{e}$ and $V^{e}$ represent the area and the volume of the element, respectively.

Remark 3: The presence of the characteristic element length $l^{e}$ in equation (56) helps us control the diffusion of the stabilized solution, avoiding overdiffusive numerical results provided that fine enough discretizations are used. This fact is clearly shown in the example of section 5.2.

\subsection{Variational form of the FIC-stabilized mass balance equa- tion}

The variational expression of the FIC-stabilized mass balance equation is obtained by multiplying Equation (55) by arbitrary test functions $v$ and integrating over the domain $\Omega$ to give

$$
\begin{aligned}
& \int_{\Omega} v \alpha \dot{\epsilon} d \Omega+\int_{\Omega} v \frac{1}{Q} \dot{p} d \Omega+\int_{\Omega} v \frac{\partial}{\partial x_{i}}\left[-\frac{1}{\mu} k_{i j}\left(\frac{\partial p}{\partial x_{j}}-\rho_{f} b_{j}\right)\right] d \Omega \\
& +\int_{\Omega} v \tau \frac{\partial}{\partial x_{i}}\left[2 G \frac{\partial \dot{\varepsilon}_{i j}}{\partial x_{j}}+\frac{\partial \dot{\sigma}^{\prime}}{\partial x_{i}}-\left(\alpha-\frac{2 G}{3 \alpha Q}\right) \frac{\partial \dot{p}}{\partial x_{i}}\right] d \Omega=0
\end{aligned}
$$

Integrating by parts the last two integrals of Equation (59) and applying the divergence theorem yields

$$
\begin{aligned}
& \int_{\Omega} v \alpha \dot{\epsilon} d \Omega+\int_{\Omega} v \frac{1}{Q} \dot{p} d \Omega-\int_{\Omega} \frac{\partial v}{\partial x_{i}}\left[-\frac{1}{\mu} k_{i j}\left(\frac{\partial p}{\partial x_{j}}-\rho_{f} b_{j}\right)\right] d \Omega \\
& +\int_{\Gamma} v\left[-\frac{1}{\mu} k_{i j}\left(\frac{\partial p}{\partial x_{j}}-\rho_{f} b_{j}\right)\right] n_{i} d \Gamma \\
& -\int_{\Omega} \tau \frac{\partial v}{\partial x_{i}}\left[2 G \frac{\partial \dot{\varepsilon}_{i j}}{\partial x_{j}}+\frac{\partial \dot{\sigma}^{\prime}}{\partial x_{i}}-\left(\alpha-\frac{2 G}{3 \alpha Q}\right) \frac{\partial \dot{p}}{\partial x_{i}}\right] d \Omega \\
& +\int_{\Gamma} v \tau\left[2 G \frac{\partial \dot{\varepsilon}_{i j}}{\partial x_{j}}+\frac{\partial \dot{\sigma}^{\prime}}{\partial x_{i}}-\left(\alpha-\frac{2 G}{3 \alpha Q}\right) \frac{\partial \dot{p}}{\partial x_{i}}\right] n_{i} d \Gamma=0
\end{aligned}
$$

where $n_{i}$ are the components of the unit normal vector to the external boundary $\Gamma$ of $\Omega$. 
Introducing the boundary condition (16) and rearranging terms we have

$$
\begin{aligned}
& \int_{\Omega} v \alpha \dot{\epsilon} d \Omega+\int_{\Omega} v \frac{1}{Q} \dot{p} d \Omega+\int_{\Omega} \frac{\partial v}{\partial x_{i}} \frac{1}{\mu} k_{i j} \frac{\partial p}{\partial x_{j}} d \Omega-\int_{\Omega} \frac{\partial v}{\partial x_{i}} \frac{1}{\mu} k_{i j} \rho_{f} b_{j} d \Omega \\
& +\int_{\Gamma} v \tilde{q}_{n} d \Gamma-\int_{\Omega} \frac{\partial v}{\partial x_{i}} \tau 2 G \frac{\partial \dot{\varepsilon}_{i j}}{\partial x_{j}} d \Omega-\int_{\Omega} \frac{\partial v}{\partial x_{i}} \tau \frac{\partial \dot{\sigma}^{\prime}}{\partial x_{i}} d \Omega \\
& +\int_{\Omega} \frac{\partial v}{\partial x_{i}} \tau\left(\alpha-\frac{2 G}{3 \alpha Q}\right) \frac{\partial \dot{p}}{\partial x_{i}} d \Omega+\int_{\Gamma} v \tau\left(2 G \frac{\partial \dot{\varepsilon}_{i j}}{\partial x_{j}} n_{i}+\frac{\partial \dot{\sigma}^{\prime}}{\partial x_{i}} n_{i}-\alpha \frac{\partial \dot{p}}{\partial x_{i}} n_{i}\right) d \Gamma \\
& +\int_{\Gamma} v \tau \frac{2 G}{3 \alpha Q} \frac{\partial \dot{p}}{\partial x_{i}} n_{i} d \Gamma=0
\end{aligned}
$$

Using the stress decomposition in (48), we can rewrite the boundary condition (14) as

$$
2 G \varepsilon_{i j} n_{j}-\frac{2 G}{3} \epsilon n_{i}+\sigma^{\prime} n_{i}-\alpha p n_{i}=\tilde{t}_{i}
$$

Next if we apply the divergence operator to both sides of Equation (62), differentiate it with respect to time and rearrange terms we have

$$
2 G \frac{\partial \dot{\varepsilon}_{i j}}{\partial x_{j}} n_{i}+\frac{\partial \dot{\sigma}^{\prime}}{\partial x_{i}} n_{i}-\alpha \frac{\partial \dot{p}}{\partial x_{i}} n_{i}=\frac{2 G}{3} \frac{\partial \dot{\epsilon}}{\partial x_{i}} n_{i}+\frac{\partial \dot{\tilde{t}}_{i}}{\partial x_{i}}
$$

In all typical problems the divergence of the traction vector will be zero and so it will be the term $\frac{\partial \dot{\tilde{t}}_{i}}{\partial x_{i}}$. Moreover, numerical tests have shown that the results are not affected by the term $\frac{2 G}{3} \frac{\partial \dot{\epsilon}}{\partial x_{i}} n_{i}$. Consequently, the stabilized mass balance equation is rewritten as

$$
\begin{aligned}
& \int_{\Omega} v \alpha \dot{\epsilon} d \Omega+\int_{\Omega} v \frac{1}{Q} \dot{p} d \Omega+\int_{\Omega} \frac{\partial v}{\partial x_{i}} \frac{1}{\mu} k_{i j} \frac{\partial p}{\partial x_{j}} d \Omega \\
& -\int_{\Omega} \frac{\partial v}{\partial x_{i}} \frac{1}{\mu} k_{i j} \rho_{f} b_{j} d \Omega+\int_{\Gamma} v \tilde{q}_{n} d \Gamma-\int_{\Omega} \frac{\partial v}{\partial x_{i}} \tau 2 G \frac{\partial \dot{\varepsilon}_{i j}}{\partial x_{j}} d \Omega \\
& -\int_{\Omega} \frac{\partial v}{\partial x_{i}} \tau \frac{\partial \dot{\sigma}^{\prime}}{\partial x_{i}} d \Omega+\int_{\Omega} \frac{\partial v}{\partial x_{i}} \tau\left(\alpha-\frac{2 G}{3 \alpha Q}\right) \frac{\partial \dot{p}}{\partial x_{i}} d \Omega \\
& +\int_{\Gamma} v \tau \frac{2 G}{3 \alpha Q} \frac{\partial \dot{p}}{\partial n} d \Gamma=0
\end{aligned}
$$

where $\frac{\partial \dot{p}}{\partial n}$ is the derivative of $\dot{p}$ in the direction of the normal to the external boundary. This derivative can be approximated as shown in Figure 2. 


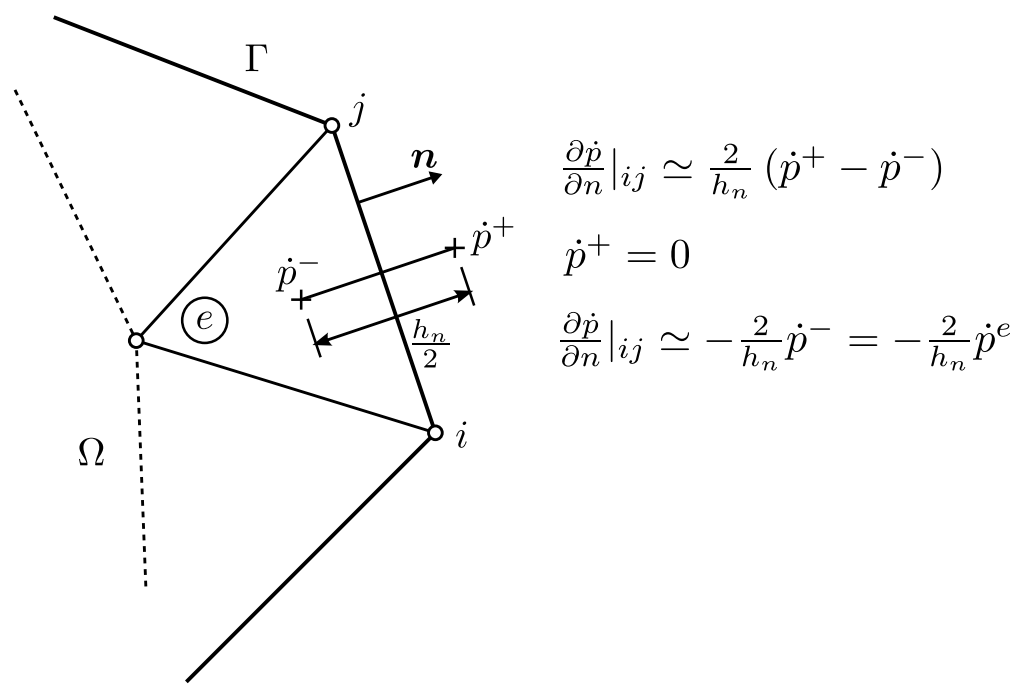

Figure 2: Computation of the term $\frac{\partial \dot{p}}{\partial n}$ at the side $i j$ of a triangle adjacent to the external boundary $\Gamma$.

Using the previous argument, the stabilized FIC form for the mass balance equation (64) finally becomes

$$
\begin{aligned}
& \int_{\Omega} v \alpha \dot{\epsilon} d \Omega+\int_{\Omega} v \frac{1}{Q} \dot{p} d \Omega+\int_{\Omega} \frac{\partial v}{\partial x_{i}} \frac{1}{\mu} k_{i j} \frac{\partial p}{\partial x_{j}} d \Omega \\
& -\int_{\Omega} \frac{\partial v}{\partial x_{i}} \frac{1}{\mu} k_{i j} \rho_{f} b_{j} d \Omega+\int_{\Gamma} v \tilde{q}_{n} d \Gamma-\int_{\Omega} \frac{\partial v}{\partial x_{i}} \tau 2 G \frac{\partial \dot{\varepsilon}_{i j}}{\partial x_{j}} d \Omega \\
& -\int_{\Omega} \frac{\partial v}{\partial x_{i}} \tau \frac{\partial \dot{\sigma}^{\prime}}{\partial x_{i}} d \Omega+\int_{\Omega} \frac{\partial v}{\partial x_{i}} \tau\left(\alpha-\frac{2 G}{3 \alpha Q}\right) \frac{\partial \dot{p}}{\partial x_{i}} d \Omega \\
& -\int_{\Gamma} v \frac{\tau}{h_{n}} \frac{4 G}{3 \alpha Q} \dot{p} d \Gamma=0
\end{aligned}
$$

where $h_{n}$ is an arbitrary distance in the normal direction to the boundary. In our work $h_{n}$ has been taken as the characteristic length $l^{e}$ of the element adjacent to the external boundary.

\section{Discretized form of the momentum and FIC- stabilized mass balance equations}

Combining the discretized form of Equation (65) along with the residual obtained from the original balance of momentum equation (24), the system of equations to be solved reads

- Balance of momentum

$$
\boldsymbol{r}_{u}=\boldsymbol{M} \ddot{\overline{\boldsymbol{u}}}+\int_{\Omega} \boldsymbol{B}^{T} \boldsymbol{\sigma}^{\prime} d \Omega-\boldsymbol{Q} \overline{\boldsymbol{p}}-\boldsymbol{f}_{u}=\mathbf{0}
$$


- Mass balance

$$
\begin{aligned}
\hat{\boldsymbol{r}}_{p}= & \left(\boldsymbol{Q}^{T}-\boldsymbol{R}\right) \dot{\overline{\boldsymbol{u}}}-\int_{\Omega}\left(\boldsymbol{\nabla} \boldsymbol{N}_{p}\right)^{T} \frac{\tau}{3} \hat{\boldsymbol{S}}^{T} \dot{\boldsymbol{\sigma}}^{\prime} d \Omega \\
& +\left(\boldsymbol{C}+\boldsymbol{T}-\boldsymbol{T}_{b}\right) \dot{\overline{\boldsymbol{p}}}+\boldsymbol{H} \overline{\boldsymbol{p}}-\boldsymbol{f}_{p}=\mathbf{0}
\end{aligned}
$$

with

$$
\begin{gathered}
\boldsymbol{R}=\int_{\Omega}\left(\boldsymbol{\nabla} \boldsymbol{N}_{p}\right)^{T} \tau 2 G \boldsymbol{S}^{T} \boldsymbol{I}_{v} \boldsymbol{B} d \Omega \\
\boldsymbol{T}=\int_{\Omega}\left(\boldsymbol{\nabla} \boldsymbol{N}_{p}\right)^{T} \tau\left(\alpha-\frac{2 G}{3 \alpha Q}\right)\left(\boldsymbol{\nabla} \boldsymbol{N}_{p}\right) d \Omega \\
\boldsymbol{T}_{b}=\int_{\Gamma} \boldsymbol{N}_{p}^{T} \frac{\tau}{h_{n}} \frac{4 G}{3 \alpha Q} \boldsymbol{N}_{p} d \Gamma
\end{gathered}
$$

where $\hat{\boldsymbol{S}}=\boldsymbol{m} \boldsymbol{\nabla}^{T}$ and the matrix $\boldsymbol{I}_{v}$ appears due to the difference between the strain tensor and the strain vector expressed in Voigt notation. For a general 3D case

$$
\boldsymbol{I}_{v}=\frac{1}{2}\left[\begin{array}{llllll}
2 & & & & & 0 \\
& 2 & & & & \\
& & 2 & & & \\
& & & 1 & & \\
& & & & 1 & \\
0 & & & & & 1
\end{array}\right]
$$

Note that since the test functions $v$ are zero on the Dirichlet boundaries $\Gamma_{p}$, matrix $\boldsymbol{T}_{b}$ must be only computed on those external boundaries where no pressure Dirichlet conditions are imposed.

Using the stabilized residual vector (67), the system of equations for a linear elastic material, quasi-static and undrained-incompressible limit case becomes

$$
\left[\begin{array}{cc}
\boldsymbol{K}_{\text {tan }} & -\boldsymbol{Q} \\
\frac{\gamma}{\beta \Delta t}\left[\boldsymbol{Q}^{T}-(\boldsymbol{R}+\boldsymbol{L})\right] & \frac{1}{\theta \Delta t} \boldsymbol{T}^{*}
\end{array}\right]_{n+1}^{i}\left[\begin{array}{l}
\delta \overline{\boldsymbol{u}} \\
\delta \overline{\boldsymbol{p}}
\end{array}\right]_{n+1}^{i+1}=-\left[\begin{array}{c}
\boldsymbol{r}_{u} \\
\hat{\boldsymbol{r}}_{p}
\end{array}\right]_{n+1}^{i}
$$

with

$$
\begin{aligned}
\boldsymbol{L} & =\int_{\Omega}\left(\boldsymbol{\nabla} \boldsymbol{N}_{p}\right)^{T} \frac{\tau}{3} \hat{\boldsymbol{S}}^{T} \boldsymbol{D} \boldsymbol{B} d \Omega \\
\boldsymbol{T}^{*} & =\int_{\Omega}\left(\boldsymbol{\nabla} \boldsymbol{N}_{p}\right)^{T} \tau \alpha\left(\boldsymbol{\nabla} \boldsymbol{N}_{p}\right) d \Omega
\end{aligned}
$$

Note that the diagonal of the iteration matrix is now different from zero. Matrices $\boldsymbol{R}$ and $\boldsymbol{L}$ emerging from the stabilized FIC formulation are essential for ensuring the consistency of the residual-based stabilization method used.

Remark 4: The consistency of residual does not directly affect the diffusion of the numerical solution, but it helps improving the condition number of 
the system matrix and facilitates the convergence of the Newton-Raphson scheme.

We highlight that the FIC-FEM formulation presented is applicable to equal order interpolation approximations of the displacements and the pressure of any degree. In this work we have implemented the FIC-FEM formulation using three-noded triangles and four-noded quadrilaterals for $2 \mathrm{D}$ problems, and four-noded tetrahedra and eight-noded hexahedra for 3D problems, with equal order interpolation for the displacements and pressure.

\section{$5 \quad$ Examples}

This section includes two academic examples designed to assess the performance of the stabilized FIC-FEM formulation in situations near the undrained-incompressible limit. The first example is an elastic soil column subjected to a surface load. The second problem is an elastic soil foundation also subjected to a surface load.

The soil column problem is analysed in a $2 \mathrm{D}$ framework under plane strain conditions, whereas the soil foundation case is tackled as a 3D problem. The problems have been solved with simple elements with equal order interpolation for the displacements and the pore pressure, using the direct (non-stabilized) formulation and the FIC-stabilized formulation presented in the previous sections. Stable mixed elements with higher order interpolation for the displacements have been used to validate the FIC-stabilized element.

In both problems, the porous medium is under totally saturated conditions with isotropic permeability. The effect of gravity is not considered.

\subsection{Elastic soil column subjected to surface loading}

This example consists on a $1 \times 30 \mathrm{~m}$ column of saturated soil subjected to a surface loading that lies on a rigid rock bed. The geometry and boundary conditions of the problem are shown in Figure 3.

The material parameters of the soil column are summarized in Table 1. It must be noticed that the indices 1 and 2 in the solid bulk modulus $K_{s}$, the fluid bulk modulus $K_{f}$ and the intrinsic permeability $k$ denote the different cases that have been considered here. Index 1 corresponds to the nearly undrained-incompressible limit, and index 2 corresponds to a more relaxed condition. 


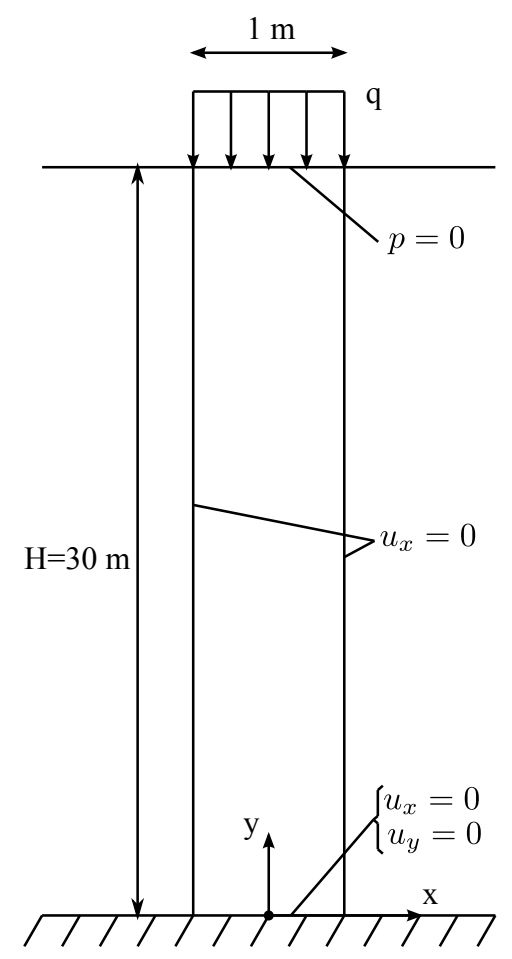

Figure 3: Elastic soil column subjected to surface loading. Geometry and boundary conditions.

\begin{tabular}{lr}
\hline Parameter & Value \\
\hline Young modulus $(E)$ & $2.5 \cdot 10^{7} \mathrm{~N} / \mathrm{m}^{2}$ \\
Poisson's ratio $(\nu)$ & 0.2 \\
Solid density $\left(\rho_{s}\right)$ & $2000 \mathrm{Kg} / \mathrm{m}^{3}$ \\
Fluid density $\left(\rho_{f}\right)$ & $1000 \mathrm{Kg} / \mathrm{m}^{3}$ \\
Porosity $(\phi)$ & 0.3 \\
Dynamic viscosity $(\mu)$ & $1 \cdot 10^{-3} \mathrm{~N} / \mathrm{m}^{2} \cdot s$ \\
\hline Solid bulk modulus 1 $\left(K_{s, 1}\right)$ & $1.5 \cdot 10^{17} \mathrm{~N} / \mathrm{m}^{2}$ \\
Fluid bulk modulus 1 $\left(K_{f, 1}\right)$ & $3 \cdot 10^{14} \mathrm{~N} / \mathrm{m}^{2}$ \\
Intrinsic Permeability $1\left(k_{1}\right)$ & $1 \cdot 10^{-14} \mathrm{~m}^{2}$ \\
\hline Solid bulk modulus 2 $\left(K_{s, 2}\right)$ & $1.5 \cdot 10^{12} \mathrm{~N} / \mathrm{m}^{2}$ \\
Fluid bulk modulus 2 $\left(K_{f, 2}\right)$ & $3 \cdot 10^{9} \mathrm{~N} / \mathrm{m}^{2}$ \\
Intrinsic Permeability $2\left(k_{2}\right)$ & $1 \cdot 10^{-11} \mathrm{~m}^{2}$ \\
\hline
\end{tabular}

Table 1: Elastic soil column problem. Material parameters.

This problem is solved in a 2D configuration under plane strain conditions. The geometry is discretized with a structured mesh of 20 quadrilat- 
eral elements. We have solved the problem using four-noded quadrilateral elements with bilinear shape functions for both the pressure and the displacements (Q4P4), and nine-noded quadrilateral elements with biquadratic shape functions for the displacements and bilinear shape functions for the pressure (Q9P4).

Two different load cases have been considered: a surface step loading (Figure 4) and a surface cyclic loading (Figure 6).

\section{Step loading case}

The main purpose of this first case is to verify that the FIC-stabilized formulation captures properly the pressure distribution along the soil column under undrained-incompressible conditions.

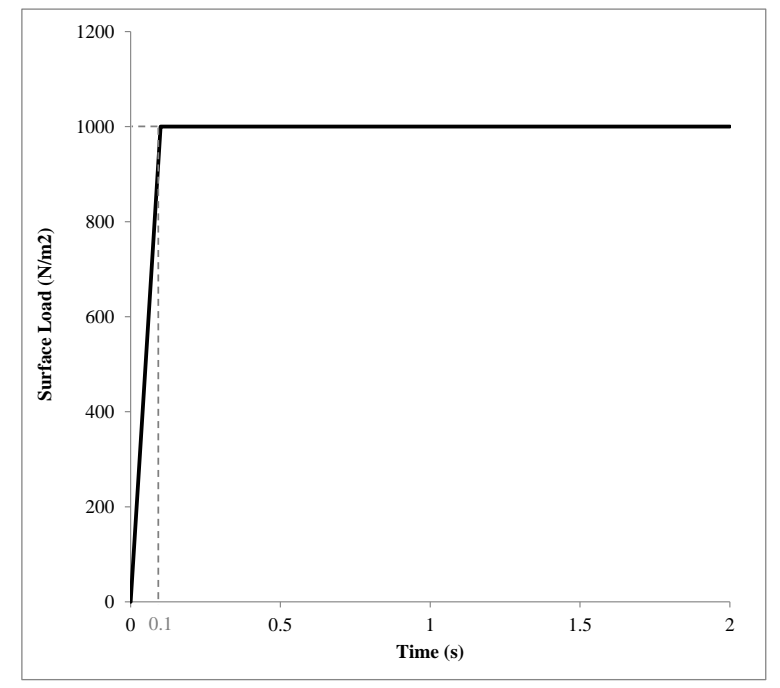

Figure 4: Surface step loading applied in the elastic soil column problem.

Figure 5 shows the normalized pore pressure along the normalized height of the soil column for nearly undrained conditions at a time $t=2 \mathrm{~s}$.

Understanding the results of Figure 5 requires taking into account the fact that gravity is not considered. Indeed, since the only force acting over the soil column is the surface load, the pressure applied by the surface load is completely transferred to the pore pressure throughout the column height and thus the ratio $p / q$ should equal 1 .

The results obtained with the non-stabilized formulation and the fournoded quadrilaterals (hereafter called Q4P4-Direct) are not able to properly capture the pressure distribution along the column. The element locks and the pressure oscillates with arbitrary values. In contrast, by using the Q4P4 element stabilized with the FIC approach presented in this work (here called 
Q4P4-FIC), the correct pressure distribution is obtained. Similar good results are obtained with the Q9P4 element, as expected.

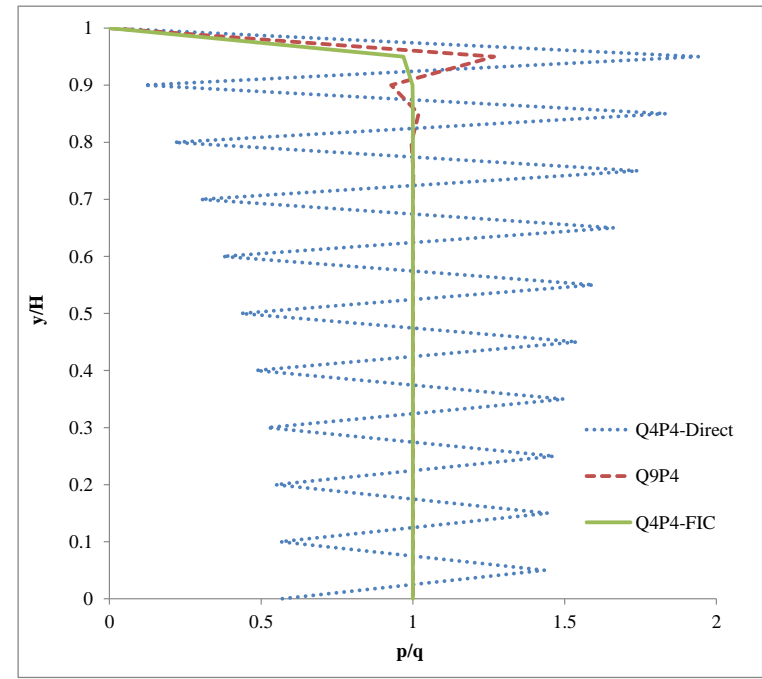

(a) Quasi-incompressible limit $\left(Q=10^{15} \mathrm{~N} / \mathrm{m}^{2}\right)$.

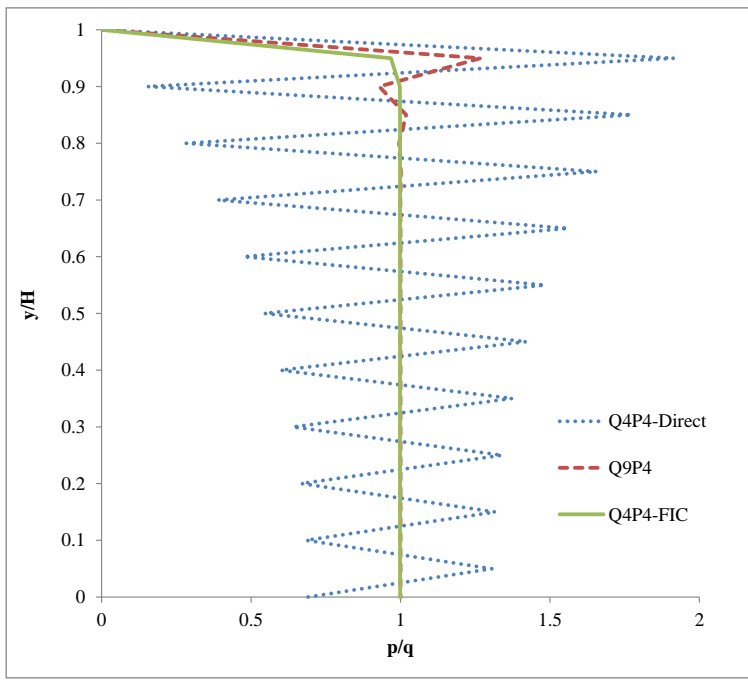

(b) Relaxed incompressibility $\left(Q=10^{10} \mathrm{~N} / \mathrm{m}^{2}\right)$.

Figure 5: Normalized pore pressure along the soil column $\left(k=1 \cdot 10^{-14} \mathrm{~m}^{2}\right.$, $\Delta t=0.02 \mathrm{~s}, t=2 \mathrm{~s})$.

Comparing the graphs in Figures 5a and 5b one can see the effect of the compressibility of the materials on the sought solution. Certainly, as we decrease the value of Biot's modulus $Q$, or equivalently, the bulk modulus of the solid and fluid phases $K_{s}$ and $K_{f}$, the amplitude of the pressure oscillations obtained with the Q4P4-Direct element decreases near the rock bed. On the other hand, the results obtained with the Q4P4-FIC and Q9P4 
elements remain unaltered.

\section{Cyclic loading case}

In this second case the objective is to analyse the evolution of the pore pressure in time, and assess the effect of the soil permeability on the dissipation of the pore pressure.

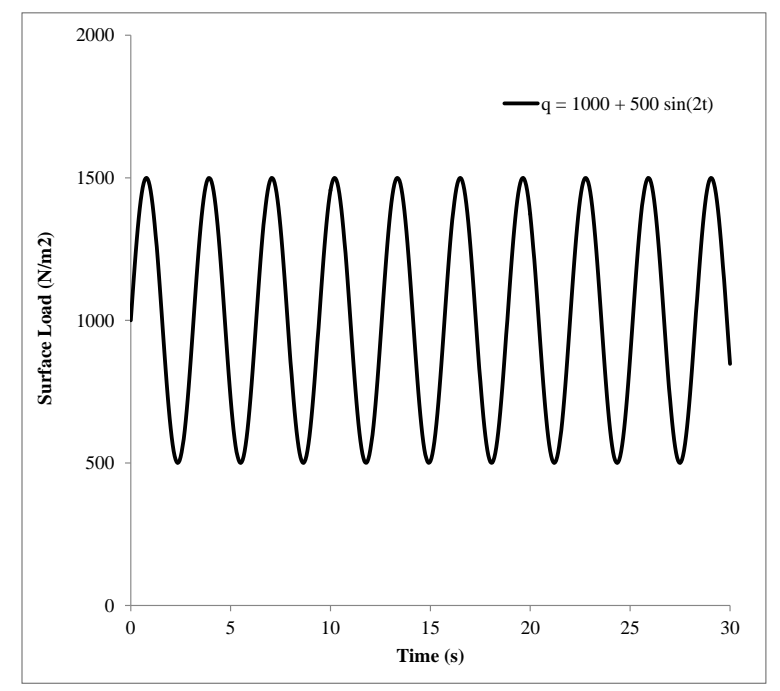

Figure 6: Surface cyclic loading applied in the elastic soil column problem.

To that purpose, Figure 7 shows the temporal evolution of the pore pressure at a node located $1.5 \mathrm{~m}$ below the surface.

Like in the previous case, the pressure obtained here should reflect the load applied on the surface. From the results in Figure 7, it is clear that the three tested elements show sinusoidal pressure evolutions in time. However, from the nearly undrained case of Figure $7 \mathrm{a}$, it appears that while the Q4P4-FIC element shows a pressure that correctly oscillates from 500 to $1500 \mathrm{~N} / \mathrm{m}^{2}$, the Q4P4-Direct element presents pressure values that vary from 1000 to $3000 \mathrm{~N} / \mathrm{m}^{2}$, making no sense. One must notice that although the Q9P4 element shows higher pressures than the stabilized element, after refining the mesh up to 60 elements, the Q9P4 results are in good agreement with the solution obtained with 20 Q4P4-FIC elements (Figure 8). This evidences the good accuracy of the stabilized FIC-FEM Q4P4 element.

Looking at the graph depicted in Figure $7 \mathrm{~b}$ one can see that, by increasing the intrinsic permeability $k$, the problem is no longer as bad conditioned as before, and so the gap in the amplitude of the three elements diminishes. Moreover, a greater permeability implies that the water can flow towards the surface due to the deformation of the column. Thereby, a dissipation in the pore pressure occurs as the computation advances in time. 


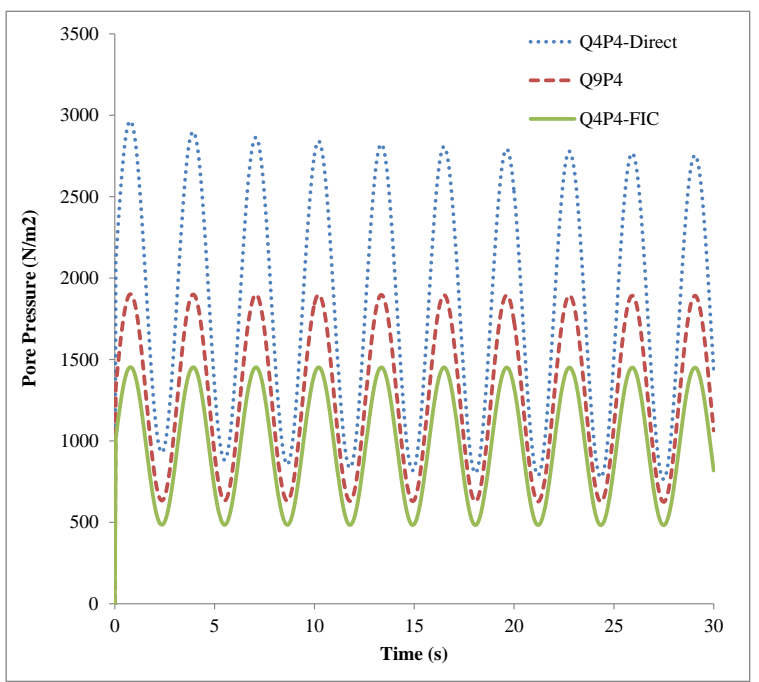

(a) Quasi-undrained limit $\left(k=10^{-14} m^{2}\right)$.

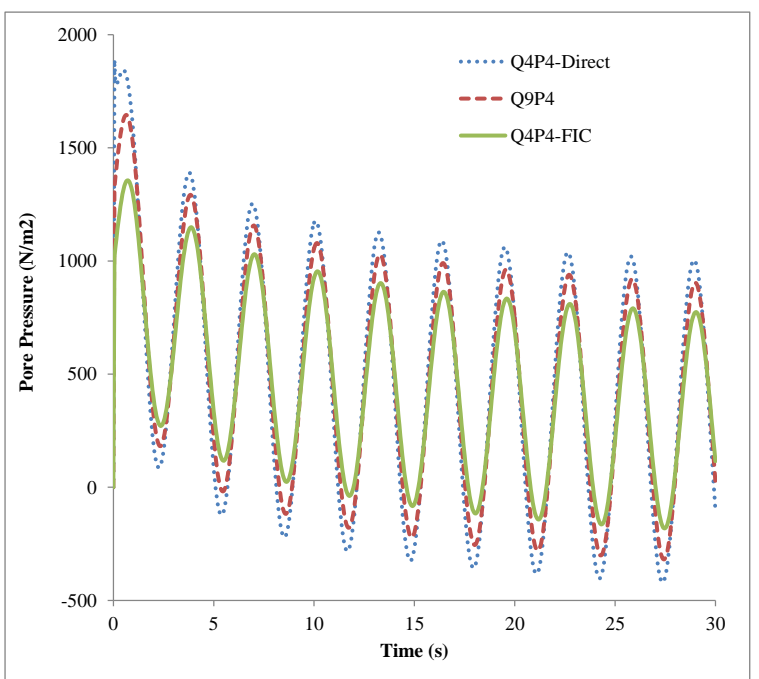

(b) Partially drained $\left(k=10^{-11} m^{2}\right)$.

Figure 7: Evolution of the pore pressure with time at a node near the surface $\left(Q=1 \cdot 10^{15} \mathrm{~N} / \mathrm{m}^{2}, \Delta t=0.05 \mathrm{~s}\right)$.

Finally, it is also interesting to notice that the pressure becomes negative after the soil has drained a certain amount of water. This is easily understood if one thinks about the elastic behaviour considered for the porous medium. Since the applied load is oscillating, the deformation of the elastic soil experiments a similar behaviour. In consequence, when the soil recovers its initial position as the load reduces, a suction appears that makes the pore pressure negative at some points near the surface. 


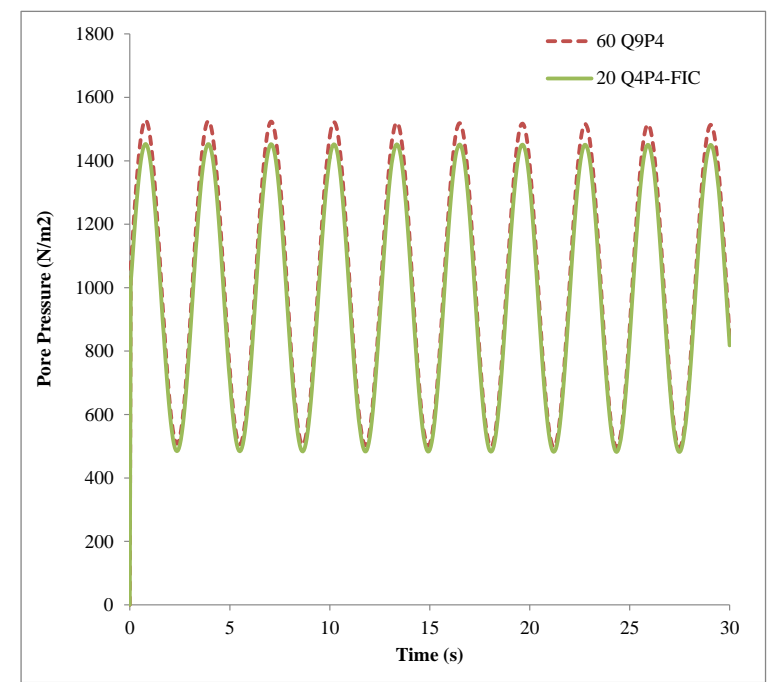

Figure 8: Time evolution of the pore pressure. Comparison between 20 Q4P4-FIC elements and 60 stable Q9P4 elements $\left(Q=1 \cdot 10^{15} \mathrm{~N} / \mathrm{m}^{2}, k=\right.$ $\left.10^{-14} \mathrm{~m}^{2}, \Delta t=0.05 \mathrm{~s}\right)$.

\subsection{Elastic soil foundation subjected to surface loading}

This second example consists on a pillar foundation on a saturated soil stratum lying over a rigid rock bed. The geometry and boundary conditions are sketched in Figure 9.

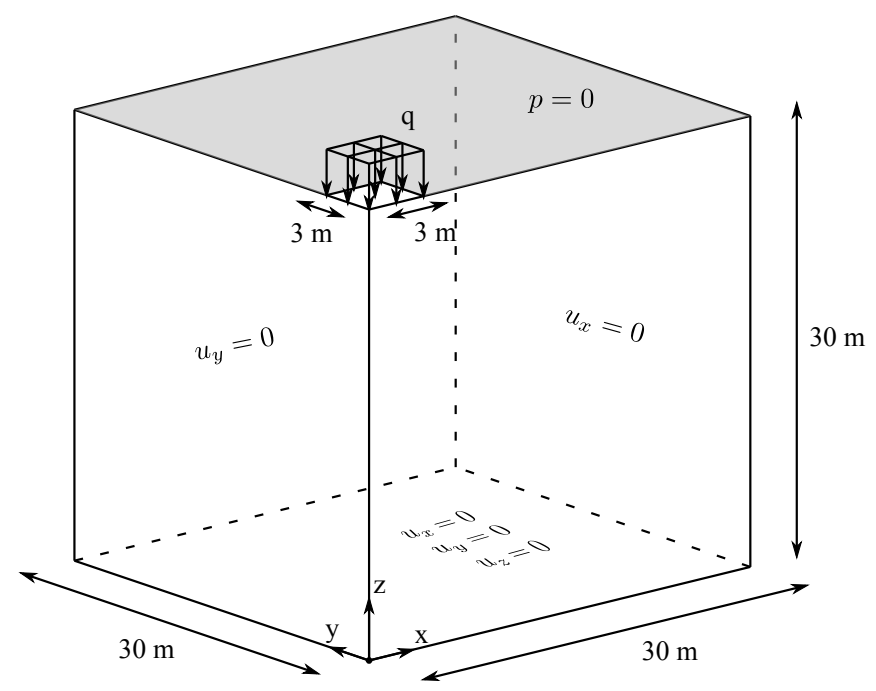

Figure 9: Elastic soil foundation subjected to surface loading. Geometry and boundary conditions. 
The material parameters for the soil are the same as for the previous example (Table 2).

\begin{tabular}{lr}
\hline Parameter & Value \\
\hline Young modulus $(E)$ & $2.5 \cdot 10^{7} \mathrm{~N} / \mathrm{m}^{2}$ \\
Poisson's ratio $(\nu)$ & 0.2 \\
Solid density $\left(\rho_{s}\right)$ & $2000 \mathrm{Kg} / \mathrm{m}^{3}$ \\
Fluid density $\left(\rho_{f}\right)$ & $1000 \mathrm{Kg} / \mathrm{m}^{3}$ \\
Porosity $(\phi)$ & 0.3 \\
Dynamic viscosity $(\mu)$ & $1 \cdot 10^{-3} \mathrm{~N} / \mathrm{m}^{2} \cdot s$ \\
\hline Solid bulk modulus 1 $\left(K_{s, 1}\right)$ & $1.5 \cdot 10^{17} \mathrm{~N} / \mathrm{m}^{2}$ \\
Fluid bulk modulus 1 $\left(K_{f, 1}\right)$ & $3 \cdot 10^{14} \mathrm{~N} / \mathrm{m}^{2}$ \\
Intrinsic Permeability $1\left(k_{1}\right)$ & $1 \cdot 10^{-14} \mathrm{~m}^{2}$ \\
\hline Solid bulk modulus 2 $\left(K_{s, 2}\right)$ & $1.5 \cdot 10^{12} \mathrm{~N} / \mathrm{m}^{2}$ \\
Fluid bulk modulus 2 $\left(K_{f, 2}\right)$ & $3 \cdot 10^{9} \mathrm{~N} / \mathrm{m}^{2}$ \\
Intrinsic Permeability $2\left(k_{2}\right)$ & $1 \cdot 10^{-11} \mathrm{~m}^{2}$ \\
\hline
\end{tabular}

Table 2: Elastic soil foundation problem. Material parameters.

In this case the soil is subjected to a surface step loading of $1 \cdot 10^{4} \mathrm{~N} / \mathrm{m}^{2}$ applied in a linear increment during $0.1 \mathrm{~s}$ (Figure 10).

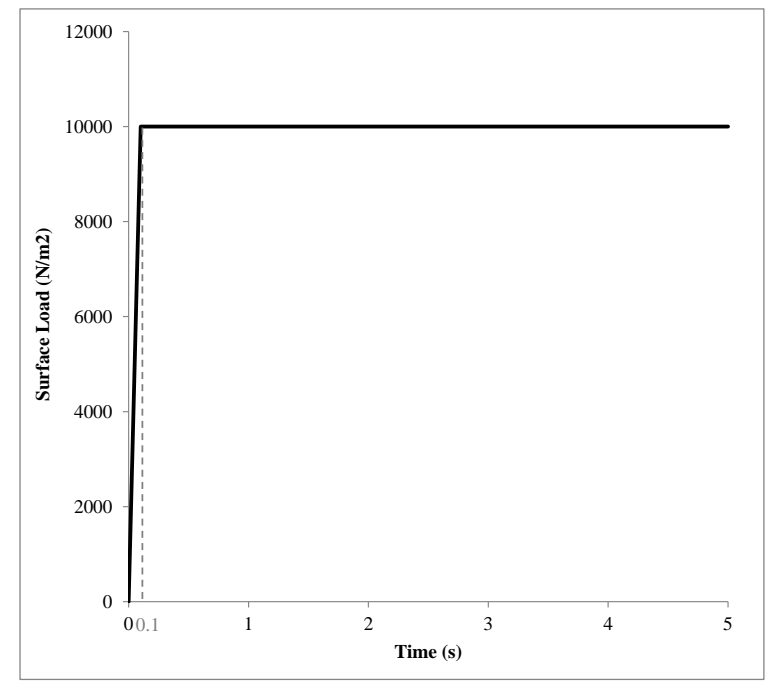

Figure 10: Surface step loading applied in the elastic soil foundation problem.

As stated before, this problem is solved by means of a 3D analysis. We use four-noded tetrahedral elements with linear interpolation for the displacements and the pressure in the non-stabilized form (T4P4-Direct) and 
the FIC-stabilized formulation (T4P4-FIC). Ten-noded stable tetrahedral elements with quadratic shape functions for the displacement field and linear shape functions for the pressure field (T10P4) have also been used in the analysis for comparison purposes.

This example has two objectives. First, it should help analysing the effect of the spatial discretization on the solution obtained with the FICstabilized formulation. Second, it may allow us to assess whether the three element types considered here converge to the expected solution when the mesh is refined.

In order to do so, two different unstructured spatial discretizations have been used: a coarse uniform mesh (Figure 11a) and a refined non-uniform mesh (Figure 11b).

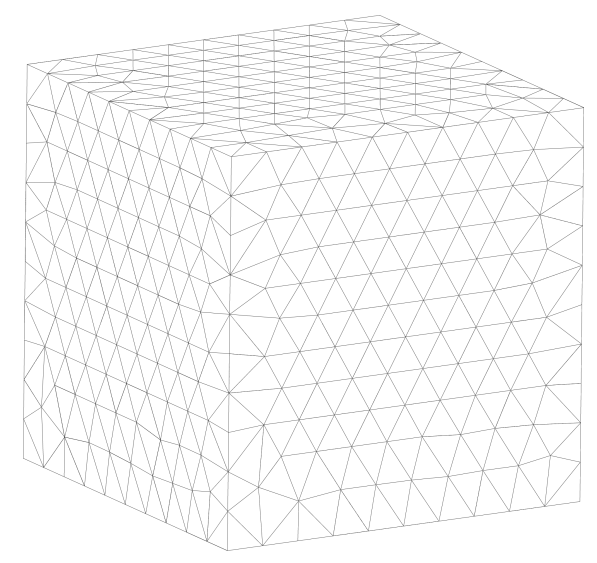

(a) Coarse uniform mesh: 9985 elements.

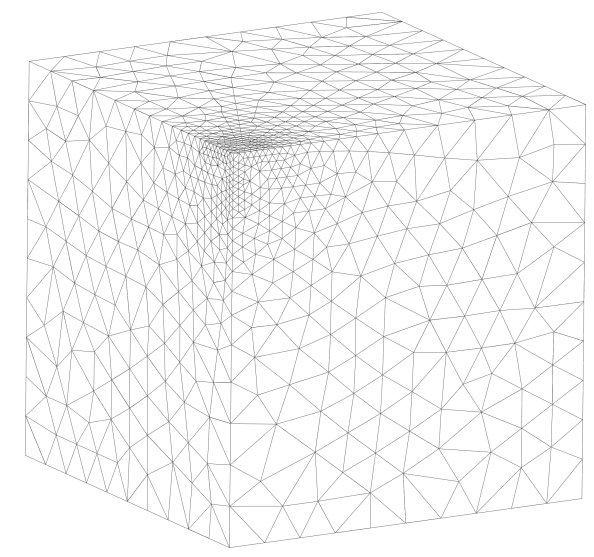

(b) Refined non-uniform mesh: 13532 elements.

Figure 11: Spatial discretizations used for the elastic soil foundation problem.

In Figure 12 we represent the evolution of the maximum pore pressure with time under nearly undrained-incompressible conditions for both the coarse and refined meshes using $\Delta t=0.02 \mathrm{~s}$. The impermeability of the case makes that the pore pressure remains constant after the first $0.1 \mathrm{~s}$ of application of the load for the two stable elements, the T10P4 and the T4P4-FIC. We see, though, a different behaviour in the unstable T4P4Direct element, showing a certain dissipation for the finer mesh.

Comparing the $\mathrm{T} 10 \mathrm{P} 4$ and the T4P4-FIC elements we notice that, while the first one surpasses the threshold of $10000 \mathrm{~N} / \mathrm{m}^{2}$ for both meshes, the latter is always below the value of the applied load. In any case, both elements approach the expected solution as the mesh is refined, which indicates a consistent response. 


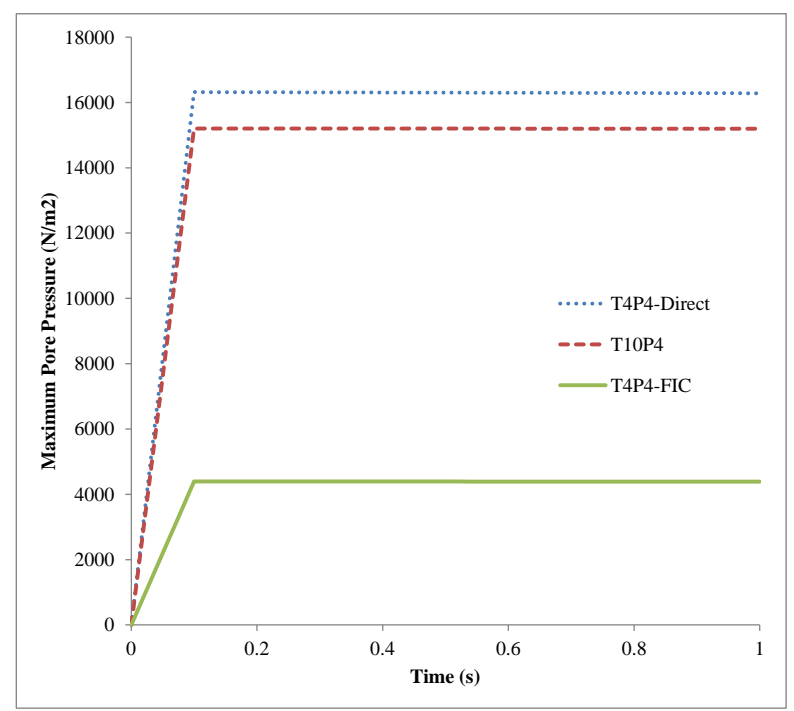

(a) Coarse mesh.

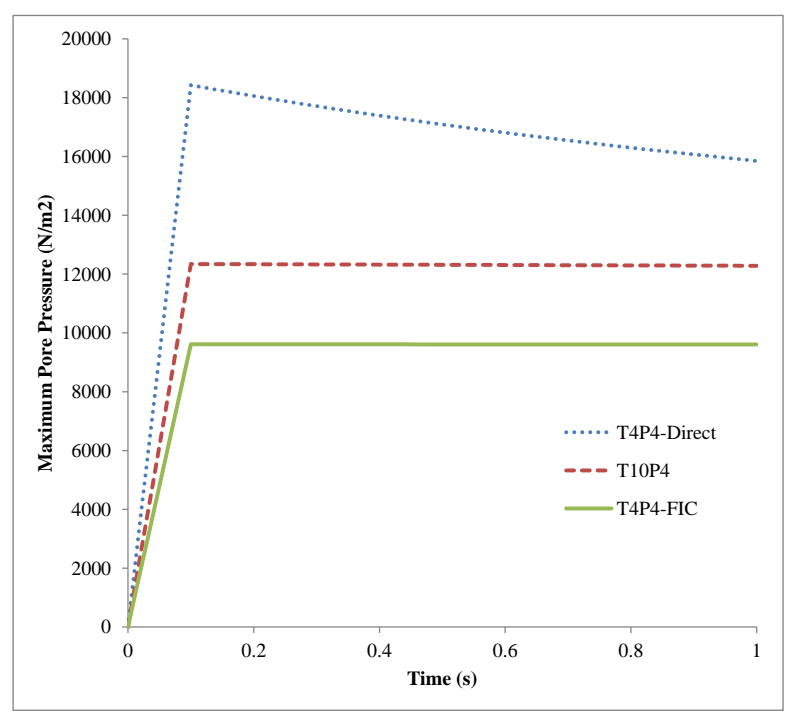

(b) Refined mesh.

Figure 12: Time evolution of the maximum pore pressure under undrainedincompressible conditions $\left(Q=1 \cdot 10^{15} \mathrm{~N} / \mathrm{m}^{2}, k=1 \cdot 10^{-14} \mathrm{~m}^{2}, \Delta t=0.02\right.$ s).

If we look at the contour lines of the pore pressure distribution obtained at time $t=1 \mathrm{~s}$ in Figure 13, we can understand the abnormal behaviour of the T4P4-Direct element. Indeed, the direct mixed formulation with equal order interpolation elements locks under undrained-incompressible conditions which leads to the incoherent pressure distribution obtained. 


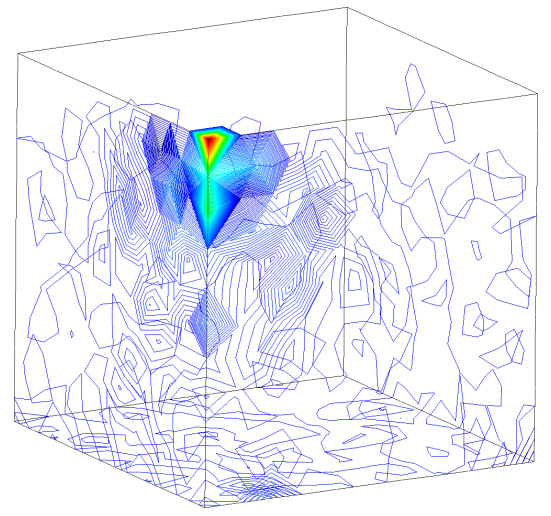

(a) T4P4-Direct elements in coarse $\operatorname{mesh}\left(p_{\max }=16282 \mathrm{~N} / \mathrm{m}^{2}\right)$.

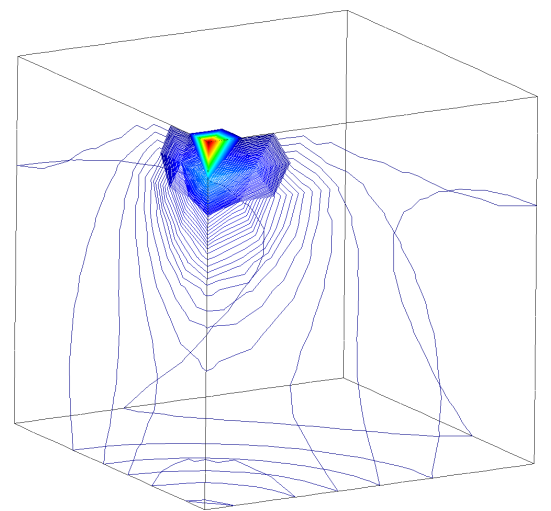

(c) T10P4 elements in coarse mesh $\left(p_{\max }=15197 \mathrm{~N} / \mathrm{m}^{2}\right)$.

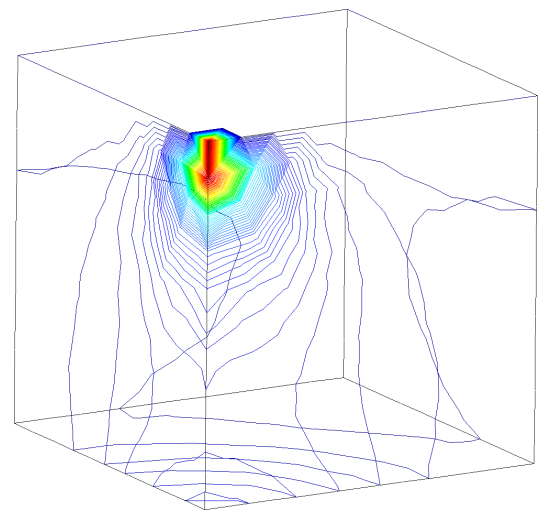

(e) T4P4-FIC elements in coarse $\operatorname{mesh}\left(p_{\max }=4390.8 \mathrm{~N} / \mathrm{m}^{2}\right)$.

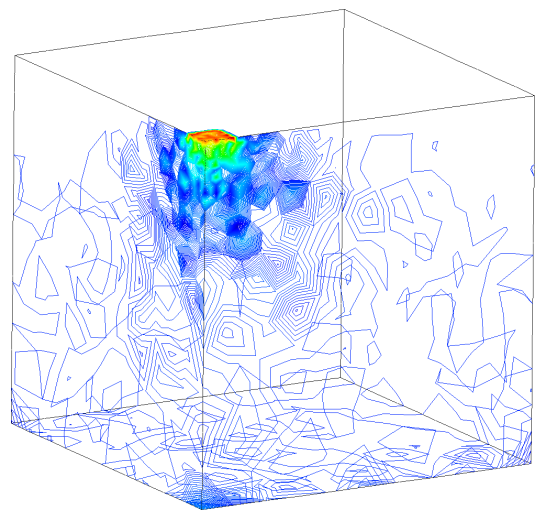

(b) T4P4-Direct elements in refined $\operatorname{mesh}\left(p_{\max }=15851 \mathrm{~N} / \mathrm{m}^{2}\right)$.

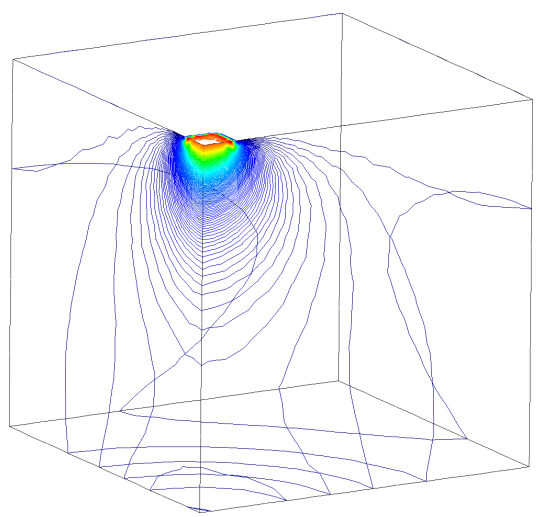

(d) T10P4 elements in refined mesh $\left(p_{\max }=12283 \mathrm{~N} / \mathrm{m}^{2}\right)$.

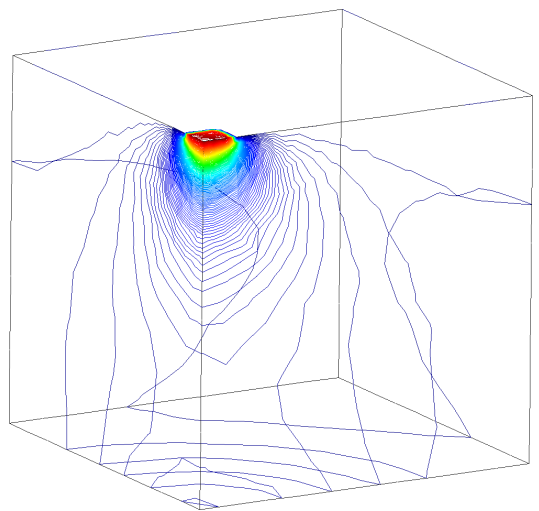

(f) T4P4-FIC elements in refined $\operatorname{mesh}\left(p_{\max }=9609.7 \mathrm{~N} / \mathrm{m}^{2}\right)$.

Figure 13: Pore pressure distribution under undrained-incompressible conditions $\left(Q=1 \cdot 10^{15} \mathrm{~N} / \mathrm{m}^{2}, k=1 \cdot 10^{-14} \mathrm{~m}^{2}, \Delta t=0.02 \mathrm{~s}, t=1 \mathrm{~s}\right)$. 
Next we evaluate the capabilities of the FIC stabilized formulation to reproduce a solution where stabilization is not needed. For this purpose the previous problem has been solved for partially drained and compressible conditions up to $t=5 \mathrm{~s}$ (Figure 14). Results are again shown at $t=1 \mathrm{~s}$ for $\Delta t=0.02 \mathrm{~s}$ (Figure 15). Under these relaxed conditions, the equal order interpolation mixed element with the direct formulation is able to capture acceptable pressure distributions as expected (Figures 15a and 15b).

The higher permeability of this second case also permits capturing the so-called Mandel-Cryer effect, i.e. the increase of pore pressure due to the application of the load is immediate, but the dissipation due to the outflow of the pore fluid is delayed by the permeability of the porous medium [51]. Figures $14 \mathrm{a}$ and $14 \mathrm{~b}$ show that, after the first $0.1 \mathrm{~s}$ of loading, the fluid starts draining due to the consolidation of the soil.

In order to properly understand the low peak pore pressure obtained with the T4P4-FIC element in Figure 14a, it is useful to recall the definition of the stabilization parameter in equation (56) and observe carefully the mesh in Figure 11a. It can be noticed that the area of application of the load is covered by only two triangular faces. This implies that the characteristic element length around that particular zone is relatively large, and so it is the stabilization parameter. As a result, one obtains an over-diffusive solution that leads to an underestimation of the maximum pore pressure.

However, it is interesting to see that the larger peak pore pressure obtained with the T4P4-Direct and the T10P4 elements, caused by an initial locking of the pressure field, converges to the solution obtained with the T4P4-FIC element after the soil consolidates.

On the other hand, the results for the maximum pressure obtained with the refined non-uniform mesh (Figures 14b, 15b, 15d and 15f) converge all to an almost identical value. This evidences that the FIC stabilization does not alter negatively the solution obtained with the original non-stabilized mixed formulation. 


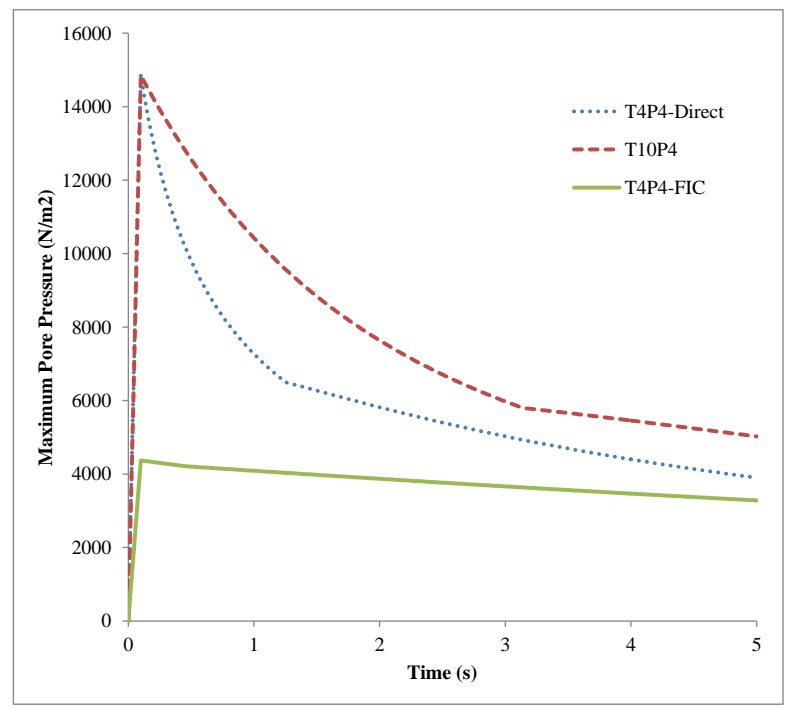

(a) Coarse mesh.

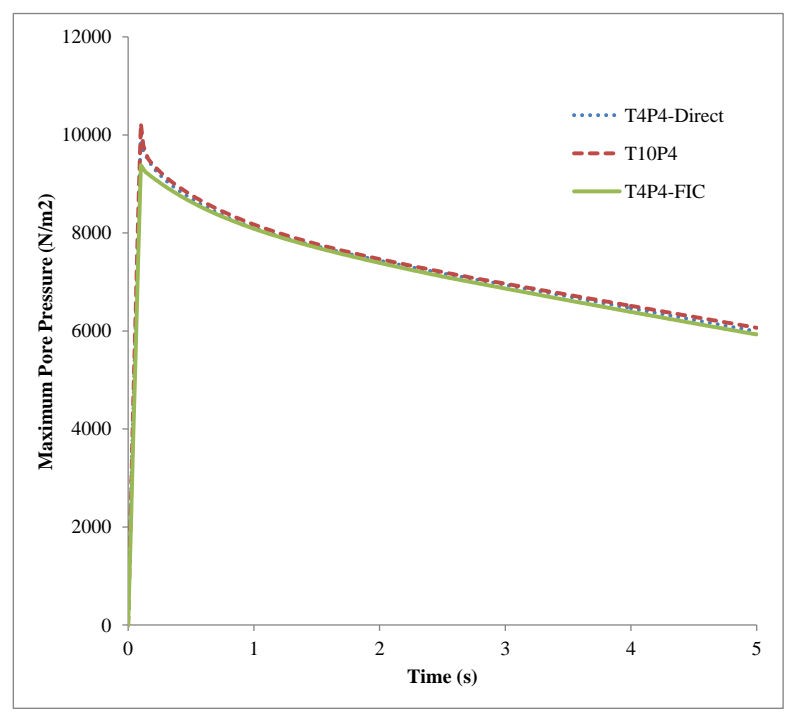

(b) Refined mesh.

Figure 14: Time evolution of the maximum pore pressure for partially compressible and drained conditions $\left(Q=1 \cdot 10^{10} \mathrm{~N} / \mathrm{m}^{2}, k=1 \cdot 10^{-11} \mathrm{~m}^{2}\right.$, $\Delta t=0.02 \mathrm{~s})$. 


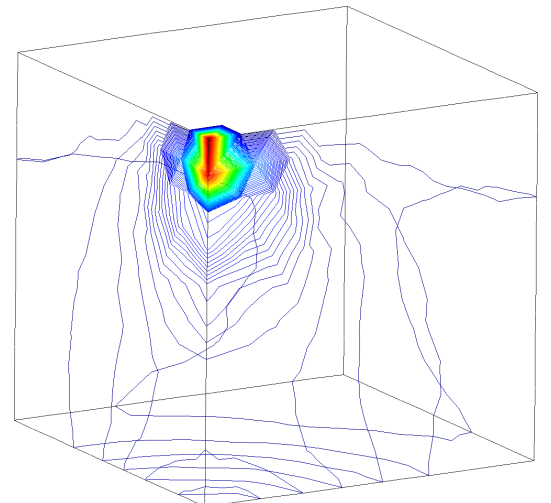

(a) T4P4-Direct elements in coarse $\operatorname{mesh}\left(p_{\max }=7268.1 \mathrm{~N} / \mathrm{m}^{2}\right)$.

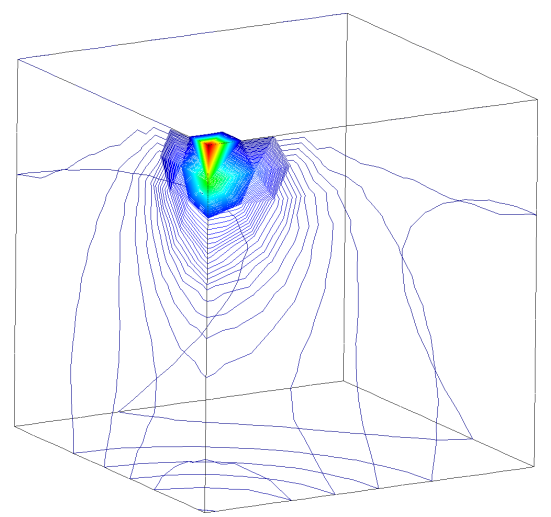

(c) T10P4 elements in coarse mesh $\left(p_{\max }=10427 \mathrm{~N} / \mathrm{m}^{2}\right)$.

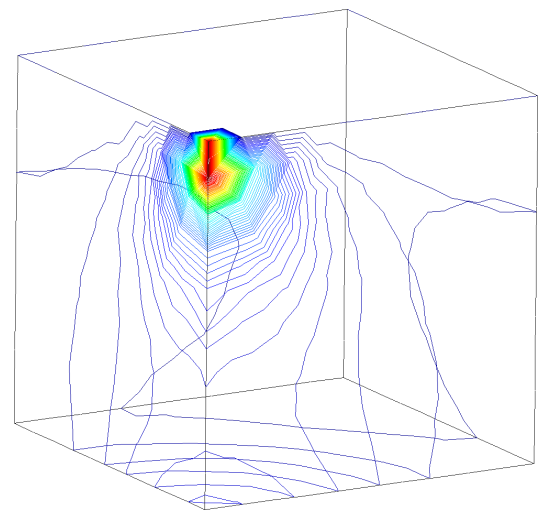

(e) T4P4-FIC elements in coarse $\operatorname{mesh}\left(p_{\max }=4089 \mathrm{~N} / \mathrm{m}^{2}\right)$.

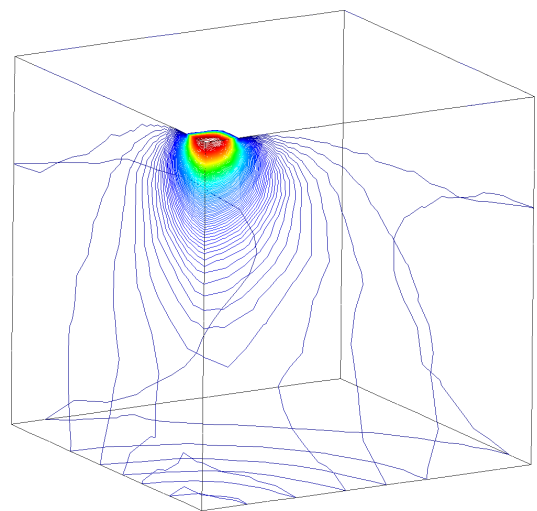

(b) T4P4-Direct elements in refined $\operatorname{mesh}\left(p_{\max }=8104.1 \mathrm{~N} / \mathrm{m}^{2}\right)$.

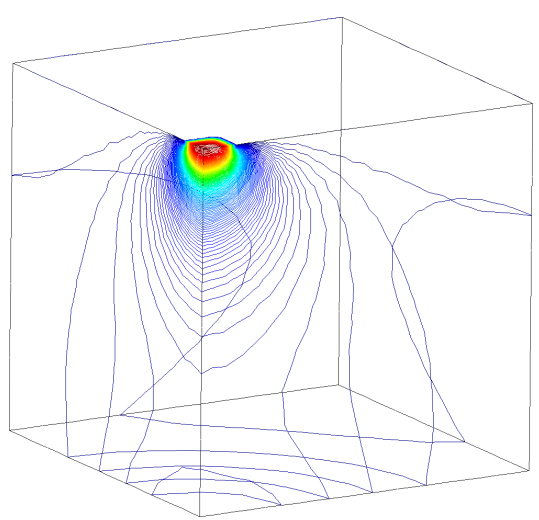

(d) T10P4 elements in refined mesh $\left(p_{\max }=8173.8 \mathrm{~N} / \mathrm{m}^{2}\right)$.

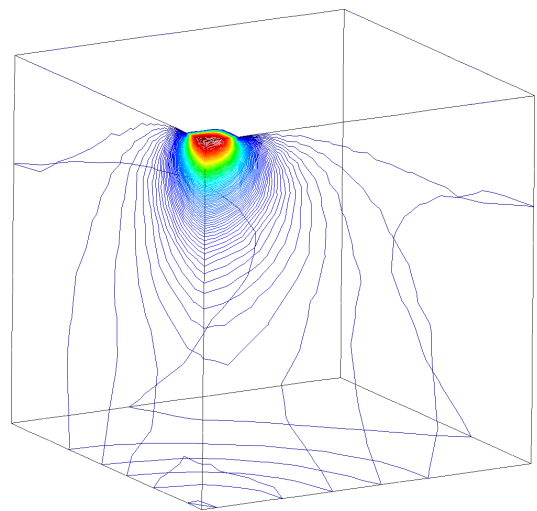

(f) T4P4-FIC elements in refined $\operatorname{mesh}\left(p_{\max }=8086.8 \mathrm{~N} / \mathrm{m}^{2}\right)$.

Figure 15: Pore pressure distribution for partially compressible and drained conditions $\left(Q=1 \cdot 10^{10} \mathrm{~N} / \mathrm{m}^{2}, k=1 \cdot 10^{-11} \mathrm{~m}^{2}, \Delta t=0.02 \mathrm{~s}, t=1 \mathrm{~s}\right)$. 


\section{Conclusions}

A stable displacement-pore pressure FIC-FEM formulation for solving coupled solid-pore fluid interaction problems has been successfully implemented for $2 \mathrm{D}$ and $3 \mathrm{D}$ analysis. The formulation represents the first application of the second order FIC mass balance equation in space to the stabilization of a poromechanics problem.

The FIC-FEM formulation has been used to solve an elastic saturated soil column subjected to surface loading in a $2 \mathrm{D}$ problem under plane strain conditions. It has proven to avoid arbitrary oscillations along the column, and it has shown consistent results for both transient and cyclic loading cases, despite modifying the material compressibility and permeability.

Additionally, the FIC-FEM procedure has been tested in a 3D problem of an elastic saturated soil foundation. The effect of the spatial discretization on the sought solution has been addressed in this case. This problem has showed that the the residual-based character of the FIC-stabilization favours that the numerical solution converges to the expected solution as the mesh is refined. Moreover, after relaxing the undrained-incompressible conditions, it has been shown that the FIC stabilization does not alter negatively the response obtained in the original non-stabilized mixed formulation, and yields an accurate solution.

Comparing this work with other classical stabilization methods, we must notice that the FIC stabilization allows solving the problem in a fully coupled manner without relying on the time stepping algorithm. It also avoids the calibration of the stabilization parameter, which simply depends on the characteristic element size.

Finally, like in the projection stabilization method, the presented FICbased approach does not require additional basis functions or element level condensation, but relies on higher-order derivatives to obtain the terms that ensure the consistency of the residual-based procedure.

\section{Acknowledgements}

This research was partially funded by the Advanced Grant project SAFECON and the Proof of Concept project FLOODSAFE of the European Research Council.

\section{References}

[1] K. Terzaghi, Theoretical soil mechanics. Wiley, New York, 1943.

[2] M. A. Biot, "General theory of three-dimensional consolidation," Journal of applied physics, vol. 12, no. 2, pp. 155-164, 1941. 
[3] B. Berkowitz, J. Bear, and C. Braester, "Continuum models for contaminant transport in fractured porous formations," Water Resources Research, vol. 24, no. 8, pp. 1225-1236, 1988.

[4] J. Adachi, E. Siebrits, A. Peirce, and J. Desroches, "Computer simulation of hydraulic fractures," International Journal of Rock Mechanics and Mining Sciences, vol. 44, no. 5, pp. 739-757, 2007.

[5] B. Carrier and S. Granet, "Numerical modeling of hydraulic fracture problem in permeable medium using cohesive zone model," Engineering fracture mechanics, vol. 79, pp. 312-328, 2012.

[6] B. Jha and R. Juanes, "Coupled modeling of multiphase flow and fault poromechanics during geologic $\mathrm{CO}_{2}$ storage," Energy Procedia, vol. 63, pp. 3313-3329, 2014.

[7] G. Zimmermann and A. Reinicke, "Hydraulic stimulation of a deep sandstone reservoir to develop an Enhanced Geothermal System: laboratory and field experiments," Geothermics, vol. 39, no. 1, pp. 70-77, 2010 .

[8] S. C. Cowin and L. Cardoso, "Interstitial flow in the hierarchical pore space architecture of bone tissue," in Poromechanics V, pp. 1203-1212, ASCE, 2013.

[9] M. A. Biot, "Theory of elasticity and consolidation for a porous anisotropic solid," Journal of applied physics, vol. 26, no. 2, pp. $182-$ $185,1955$.

[10] M. A. Biot, "Theory of propagation of elastic waves in a fluid-saturated porous solid. I: Low-frequency range. II: Higher frequency range," the Journal of the Acoustical Society of America, vol. 28, no. 2, pp. 168-191, 1956.

[11] J. Ghaboussi and E. L. Wilson, "Flow of compressible fluid in porous elastic media," International Journal for Numerical Methods in Engineering, vol. 5, no. 3, pp. 419-442, 1973.

[12] O. C. Zienkiewicz, C. T. Chang, and P. Bettess, "Drained, undrained, consolidating and dynamic behaviour assumptions in soils," Géotechnique, vol. 30, no. 4, pp. 385-395, 1980.

[13] O. C. Zienkiewicz and P. Bettess, "Soils and other saturated media under transient, dynamic conditions: general formulation and the validity of various simplifying assumptions," 1982.

[14] O. C. Zienkiewicz, "Basic formulation of static and dynamic behaviour of soil and other porous media," in Numerical methods in geomechanics, pp. 39-55, Springer, 1982. 
[15] O. C. Zienkiewicz and T. Shiomi, "Dynamic behaviour of saturated porous media; The generalized Biot formulation and its numerical solution," International journal for numerical and analytical methods in geomechanics, vol. 8, no. 1, pp. 71-96, 1984.

[16] O. C. Zienkiewicz, A. H. C. Chan, M. Pastor, D. K. Paul, and T. Shiomi, "Static and dynamic behaviour of soils: a rational approach to quantitative solutions. I. Fully saturated problems," in Proceedings of the Royal Society of London, vol. 429, pp. 285-309, The Royal Society, 1990.

[17] O. C. Zienkiewicz, Y. M. Xie, B. A. Schrefler, A. Ledesma, and N. Bicanic, "Static and dynamic behaviour of soils: a rational approach to quantitative solutions. II. Semi-saturated problems," in Proceedings of the Royal Society of London, vol. 429, pp. 311-321, The Royal Society, 1990.

[18] D. Gawin and B. A. Schrefler, "Thermo-hydro-mechanical analysis of partially saturated porous materials," Engineering Computations, vol. 13 , no. 7 , pp. 113-143, 1996.

[19] A. R. Khoei, A. R. Azami, and S. M. Haeri, "Implementation of plasticity based models in dynamic analysis of earth and rockfill dams: A comparison of Pastor-Zienkiewicz and cap models," Computers and Geotechnics, vol. 31, pp. 385-410, 2004.

[20] I. Babuška, "The finite element method with lagrangian multipliers," Numerische Mathematik, vol. 20, no. 3, pp. 179-192, 1973.

[21] F. Brezzi, "On the existence, uniqueness and approximation of saddle-point problems arising from lagrangian multipliers," Revue française d'automatique, informatique, recherche opérationnelle. Analyse numérique, vol. 8, no. 2, pp. 129-151, 1974.

[22] A. J. Chorin, "A numerical method for solving incompressible viscous flow problems," Journal of Computational Physics, vol. 2, no. 1, pp. 12$26,1967$.

[23] F. Brezzi and J. Pitkäranta, "On the stabilization of finite element approximations of the Stokes equations," in Efficient Solutions of Elliptic Systems (W. Hackbusch, ed.), vol. 10 of Notes on Numerical Fluid Mechanics, pp. 11-19, Vieweg, Wiesbaden, 1984.

[24] T. J. R. Hughes, L. P. Franca, and M. Balestra, "A new finite element formulation for computational fluid dynamics: V. Circumventing the Babuška-Brezzi condition: A stable Petrov-Galerkin formulation of the Stokes problem accommodating equal-order interpolations," Computer 
Methods in Applied Mechanics and Engineering, vol. 59, pp. 85-99, 1986.

[25] O. C. Zienkiewicz and R. Codina, "A general algorithm for compressible and incompressible flow. Part I. The split, characteristic-based scheme," International Journal for Numerical Methods in Fluids, vol. 20, no. 8-9, pp. 869-885, 1995.

[26] E. Oñate, "Derivation of stabilized equations for numerical solution of advective-diffusive transport and fluid flow problems," Computer Methods in Applied Mechanics and Engineering, vol. 151, no. 1, pp. 233-265, 1998.

[27] E. Oñate, "A stabilized finite element method for incompressible viscous flows using a finite increment calculus formulation," Computer Methods in Applied Mechanics and Engineering, vol. 182, no. 3, pp. 355-370, 2000 .

[28] M. Pastor, M. Quecedo, and O. C. Zienkiewicz, "A mixed displacementpressure formulation for numerical analysis of plastic failure," Computers ES Structures, vol. 62, no. 1, pp. 13-23, 1997.

[29] O. C. Zienkiewicz, J. Rojek, R. L. Taylor, and M. Pastor, "Triangles and tetrahedra in explicit dynamic codes for solids," International Journal for Numerical Methods in Engineering, vol. 43, no. 3, pp. 565-583, 1998.

[30] M. Chiumenti, Q. Valverde, C. A. De Saracibar, and M. Cervera, "A stabilized formulation for incompressible elasticity using linear displacement and pressure interpolations," Computer Methods in Applied Mechanics and Engineering, vol. 191, no. 46, pp. 5253-5264, 2002.

[31] E. Oñate, R. L. Taylor, O. C. Zienkiewicz, and J. Rojek, "A residual correction method based on finite calculus," Engineering Computations, vol. 20, no. 5/6, pp. 629-658, 2003.

[32] E. Oñate, J. Rojek, R. L. Taylor, and O. C. Zienkiewicz, "Finite calculus formulation for incompressible solids using linear triangles and tetrahedra," International Journal for Numerical Methods in Engineering, vol. 59, no. 11, pp. 1473-1500, 2004.

[33] O. C. Zienkiewicz, M. Huang, and M. Pastor, "Computational soil dynamics - A new algorithm for drained and undrained conditions," in Computer Methods and Advances in Geomechanics (H. J. Siriwardane and M. M. Zaman, eds.), pp. 47-59, Balkema, Rotterdam, 1994.

[34] M. Pastor, O. C. Zienkiewicz, T. L. L. Xiaoqing, and M. Huang, "Stabilized finite elements with equal order of interpolation for soil dynamics 
problems," Archives of Computational Methods in Engineering, vol. 6, no. 1, pp. 3-33, 1999.

[35] M. Huang, S. Wu, and O. C. Zienkiewicz, "Incompressible or nearly incompressible soil dynamic behaviour-a new staggered algorithm to circumvent restrictions of mixed formulation," Soil Dynamics and Earthquake Engineering, vol. 21, no. 2, pp. 169-179, 2001.

[36] A. Masud and T. J. R. Hughes, "A stabilized mixed finite element method for Darcy flow," Computer Methods in Applied Mechanics and Engineering, vol. 191, no. 39, pp. 4341-4370, 2002.

[37] J. A. White and R. I. Borja, "Stabilized low-order finite elements for coupled solid-deformation/fluid-diffusion and their application to fault zone transients," Computer Methods in Applied Mechanics and Engineering, vol. 197, no. 49, pp. 4353-4366, 2008.

[38] W. Sun, J. T. Ostien, and A. G. Salinger, "A stabilized assumed deformation gradient finite element formulation for strongly coupled poromechanical simulations at finite strain," International Journal for Numerical and Analytical Methods in Geomechanics, vol. 37, no. 16, pp. 27552788, 2013.

[39] J. Choo and R. I. Borja, "Stabilized mixed finite elements for deformable porous media with double porosity," Computer Methods in Applied Mechanics and Engineering, vol. 293, pp. 131-154, 2015.

[40] W. Sun, "A stabilized finite element formulation for monolithic thermohydro-mechanical simulations at finite strain," International Journal for Numerical Methods in Engineering, vol. 103, no. 11, pp. 798-839, 2015 .

[41] E. Oñate, S. R. Idelsohn, and C. Felippa, "Consistent pressure laplacian stabilization for incompressible continua via higher-order finite calculus," International Journal for Numerical Methods in Engineering, vol. 87, no. 1-5, pp. 171-195, 2011.

[42] E. Oñate, A. Franci, and J. M. Carbonell, "Lagrangian formulation for finite element analysis of quasi-incompressible fluids with reduced mass losses," International Journal for Numerical Methods in Fluids, vol. 74, no. 10, pp. 699-731, 2014.

[43] M. Preisig and J. H. Prévost, "Stabilization procedures in coupled poromechanics problems: A critical assessment," International Journal for Numerical and Analytical Methods in Geomechanics, vol. 35, no. 11, pp. 1207-1225, 2011. 
[44] M. A. Biot and D. G. Willis, "The elastic coefficients of the theory of consolidation," Journal of applied mechanics, vol. 24, pp. 594-601, 1957.

[45] B. G. Galerkin, "Series solution of some problems of elastic equilibrium of rods and plates," Vestnik Inzhenerov $i$ Tekhnikov, vol. 19, no. 7, pp. 897-908, 1915.

[46] C. B. Biezeno and R. Grammel, Technische Dynamik. Springer-Verlag, 1939.

[47] N. M. Newmark, "A method of computation for structural dynamics," Journal of the Engineering Mechanics Division, vol. 85, no. 3, pp. 6794, 1959.

[48] W. J. T. Daniel, "Modal methods in finite element fluid-structure eigenvalue problems," International Journal for Numerical Methods in Engineering, vol. 15, no. 8, pp. 1161-1175, 1980.

[49] O. C. Zienkiewicz, S. Qu, R. L. Taylor, and S. Nakazawa, "The patch test for mixed formulations," International Journal for Numerical Methods in Engineering, vol. 23, no. 10, pp. 1873-1883, 1986.

[50] O. C. Zienkiewicz, R. L. Taylor, and J. Z. Zhu, The Finite Element Method, vol. 1. Butterworth-Heinemann, 6th ed., 2005.

[51] A. Verruijt, Theory and problems of poroelasticity. Delft University of Technology, 2013. 